\title{
Odd entanglement entropy and logarithmic negativity for thermofield double states
}

\author{
Mostafa Ghasemi, $^{a}$ Ali Naseh ${ }^{a}$ and Reza Pirmoradian ${ }^{a, b}$ \\ ${ }^{a}$ School of Particles and Accelerators, Institute for Research in Fundamental Sciences (IPM), \\ P.O. Box 19395-5531, Tehran, Iran \\ ${ }^{b}$ Department of physics, Islamic Azad University Central Tehran Branch, \\ Tehran, Iran \\ E-mail: ghasemi.mg@ipm.ir, naseh@ipm.ir, rezapirmoradian@ipm.ir
}

AbStRaCT: We investigate the time evolution of odd entanglement entropy (OEE) and logarithmic negativity (LN) for the thermofield double (TFD) states in free scalar quantum field theories using the covariance matrix approach. To have mixed states, we choose noncomplementary subsystems, either adjacent or disjoint intervals on each side of the TFD. We find that the time evolution pattern of OEE is a linear growth followed by saturation. On a circular lattice, for longer times the finite size effect demonstrates itself as oscillatory behavior. In the limit of vanishing mass, for a subsystem containing a single degree of freedom on each side of the TFD, we analytically find the effect of zero-mode on the time evolution of $\mathrm{OEE}$ which leads to logarithmic growth in the intermediate times. Moreover, for adjacent intervals we find that the $\mathrm{LN}$ is zero for times $t<\beta / 2$ (half of the inverse temperature) and after that, it begins to grow linearly. For disjoint intervals at fixed temperature, the vanishing of $\mathrm{LN}$ is observed for times $t<d / 2$ (half of the distance between intervals). We also find a similar delay to see linear growth of $\Delta S=S_{\mathrm{OEE}}-S_{\mathrm{EE}}$. All these results show that the dynamics of these measures are consistent with the quasi-particle picture, of course apart from the logarithmic growth.

Keywords: AdS-CFT Correspondence, Conformal Field Theory, Field Theories in Lower Dimensions

ArXiv EPrint: 2106.15451 


\section{Contents}

1 Introduction 1

2 Covariance matrix for Gaussian TFD state 5

2.1 Free real scalar QFT on a lattice 6

$\begin{array}{lll}2.2 & \text { TFD state for a simple harmonic oscillator } & 7\end{array}$

2.3 Covariance matrix formalism 8

2.3.1 Covariance matrix for TFD state of the harmonic oscillator $\quad 10$

2.3.2 Covariance matrix for TFD state of real scalar QFT 11

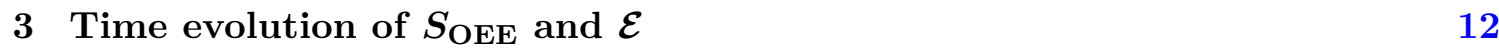

4 Numerical results $\quad \mathbf{1 5}$

$\begin{array}{lll}4.1 \text { Adjacent intervals on each side } & 16\end{array}$

$\begin{array}{lll}4.2 & \text { Disjoint intervals on each side } & 17\end{array}$

$\begin{array}{lll}4.3 & \text { Different quasi-particles and different information quantities } & 21\end{array}$

4.4 LN vs mutual and 1/2-Rényi mutual information 22

4.5 Some inequalities for OEE 23

5 Logarithmic growth at intermediate times $\quad 23$

6 Conclusion 28

A Temperature effects: various entanglement measures 32

A.1 Two adjacent intervals on each side $\quad 32$

A.2 Two disjoint intervals on each side 34

B Mass effects: various entanglement measures $\quad 34$

$\begin{array}{ll}\text { C Zero-mode computations } & 36\end{array}$

\section{Introduction}

Understanding the non-equilibrium dynamics of the isolated many-body quantum systems has been one of the major research avenues, both theoretical and experimental, in the last few decades [1-5]. One of the protocols for preparing the out of equilibrium systems is the global quench setting that enables us to study thermalization in isolated quantum systems and can be best understood by phenomenological quasi-particle picture [6-10]. In this setup, an isolated system is initially prepared at $t=0$ in a given ground state $\left|\psi_{0}\right\rangle$ of a certain Hamiltonian $H_{0}$ and undergoes a sudden change in a parameter of the Hamiltonian 
at $t=0$. The system evolves with a new Hamiltonian $H$ in such a way that $\left[H, H_{0}\right] \neq 0$ and the time evolved state at time $t$ is given by $|\psi(t)\rangle=e^{-i H t}\left|\psi_{0}\right\rangle$. Since the evolution is unitary, the final state of the system is described by a pure state and one of the appropriate concepts for understanding the thermalization is quantum entanglement, $S_{\mathrm{EE}}$ [11]. When considering this time evolution, there is a regime in which the initial growth is linear and it occurs when the lattice spacing $\delta$ is much smaller than the correlation length, $\beta$, which itself is much smaller than the subsystem size $l$, i.e.,

$$
\delta \ll \beta \ll l \text {. }
$$

In the quasi-particle picture, the quench creates independent entangled pairs which propagate on a circle (with circumference $\mathcal{L}$ ) in a ballistic fashion with an effective velocity

$$
v_{n}=\frac{\mathcal{L}}{2 \pi} \partial_{n} \omega_{n}
$$

where $n$ is an integer that runs from 0 to the total number of lattice sites and $\omega_{n}$ is given by (2.6). The upper bound of velocity is known as Lieb-Robinson bound [12]. When the quasi-particles and their partners laying in the interval and it is complementary, respectively(or vise versa), we observe the linear growth in the entanglement entropy where Alba and Calabrese $[9,10]$ provided a formula for it. By carefully tracking quasiparticles on a circle leaving and re-entering the interval, a phenomenological relation for $S_{\mathrm{EE}}^{\mathrm{TFD}}(t)-S_{\mathrm{EE}}^{\mathrm{TFD}}(t=0)$ in TFD state, ${ }^{1}$ is proposed $[9,10,13]$

$$
\begin{aligned}
S_{\mathrm{EE}}^{\mathrm{TFD}}(t)- & S_{\mathrm{EE}}^{\mathrm{TFD}}(t=0)= \\
\sum_{n} \mathbf{s}_{n}^{\mathrm{TFD}} \mathcal{L} \operatorname{frac}\left(\frac{v_{n} t}{\mathcal{L}}\right) & \text { if } \mathcal{L} \operatorname{frac}\left(\frac{v_{n} t}{\mathcal{L}}\right)<l, \\
\sum_{n} \mathbf{s}_{n}^{\mathrm{TFD}} l & \text { if } l \leq \mathcal{L} \operatorname{frac}\left(\frac{v_{n} t}{\mathcal{L}}\right)<\mathcal{L}-l, \\
\sum_{n} \mathbf{s}_{n}^{\mathrm{TFD}} \mathcal{L}\left(1-\operatorname{frac}\left(\frac{v_{n} t}{\mathcal{L}}\right)\right) & \text { if } \mathcal{L}-l \leq \mathcal{L} \operatorname{frac}\left(\frac{v_{n} t}{\mathcal{L}}\right),
\end{aligned}
$$

where frac denotes the fractional part and $\mathbf{s}_{n}^{\mathrm{TFD}}$ is $^{2}$

$$
\mathbf{s}_{n}^{\mathrm{TFD}}=\frac{2}{\mathcal{L}}\left(\frac{\beta \omega_{n}}{e^{\beta \omega_{n}}-1}-\log \left(1-e^{-\beta \omega_{n}}\right)\right)
$$

However, the entanglement entropy is only a proper measure to capture the dynamics of entanglement for pure quantum states and to have a deeper insight into the spreading of information in the out of equilibrium situations, we need to investigate the dynamics of the mixed states correlation measures. One of those measures is mutual information (MI) which is defined for disjoint subregions $A$ and $B$. It is given as a linear combination of entanglement entropies of that regions,

$$
I(A, B)=S_{\mathrm{EE}, A}+S_{\mathrm{EE}, B}-S_{\mathrm{EE}, A \cup B}
$$

\footnotetext{
${ }^{1}$ In the following we will define this state.

${ }^{2}$ The part within the large parentheses represents a contribution to the thermodynamic entropy of the free boson system at the inverse temperature $\beta$ from the mode $n$.
} 
However, MI includes both classical and quantum correlations between the subregions $A$ and $B$ and since it does not vanish for separable states it would not be a good measure of entanglement [14]. Besides the MI, in the quantum information context, there are various measures for capturing the entanglement dynamics of mixed states. But, these measures are usually based on optimization procedures [11], which are intractable approaches and we need a way to have explicit computational prescriptions. Among all these proposed entanglement measures for mixed states, the logarithmic negativity (LN) is the best computable measure which is expected to capture only quantum correlations [15-17]. Recently, another information theoretic quantity is introduced which is called the odd entanglement entropy (OEE) [18]. In the context of the AdS/CFT duality [19], the LN [20, 21] and OEE [18] are dual to the same geometric object which is called the entanglement wedge cross-section (EWC) $[22,23]$. The dynamics of EWC has been recently studied in [24-27] and the authors of $[28,29]$ have denoted that these two measures are proportional in the case of integrable systems.

The LN and OEE are defined by taking partial transposing of the state which it is obtained as follows. Let $\left|e_{i}^{(1)}\right\rangle$ and $\left|e_{i}^{(2)}\right\rangle$ denote the orthogonal basis for states of $\mathcal{H}_{A_{1}}$ and $\mathcal{H}_{A_{2}}$, respectively. A density matrix acting on the bipartite Hilbert space $\mathcal{H}=\mathcal{H}_{A_{1}} \otimes \mathcal{H}_{A_{2}}$, with the basis $\left|e_{i}^{(1)} e_{i}^{(2)}\right\rangle=\left|e_{i}^{(1)}\right\rangle \otimes\left|e_{i}^{(2)}\right\rangle$, is denoted by $\rho_{A_{1} A_{2}}$. The $\rho_{A_{1} A_{2}}$ can be expanded in a basis $\left|e_{i}^{(1)} e_{j}^{(2)}\right\rangle$ of $\mathcal{H}$ as follows

$$
\rho_{A_{1} A_{2}}=\sum_{i j k l}\left\langle e_{i}^{(1)} e_{j}^{(2)}\left|\rho_{A_{1} A_{2}}\right| e_{k}^{(1)} e_{l}^{(2)}\right\rangle\left|e_{i}^{(1)} e_{j}^{(2)}\right\rangle\left\langle e_{k}^{(1)} e_{l}^{(2)}\right|
$$

The partial transposition of the density matrix $\rho_{A_{1} A_{2}}$ with respect to the subsystem $A_{2}$ is given by swapping the matrix elements in the subsystem $A_{2}$,

$$
\left\langle e_{i}^{(1)} e_{j}^{(2)}\left|\rho_{A_{1} A_{2}}^{T_{A_{2}}}\right| e_{k}^{(1)} e_{l}^{(2)}\right\rangle=\left\langle e_{i}^{(1)} e_{l}^{(2)}\left|\rho_{A_{1} A_{2}}\right| e_{k}^{(1)} e_{j}^{(2)}\right\rangle .
$$

Accordingly, with respect to the original basis of $\mathcal{H}$ one has

$$
\rho_{A_{1} A_{2}}^{T_{A_{2}}}=\sum_{i j k l}\left\langle e_{i}^{(1)} e_{l}^{(2)}\left|\rho_{A_{1} A_{2}}\right| e_{k}^{(1)} e_{j}^{(2)}\right\rangle\left|e_{i}^{(1)} e_{j}^{(2)}\right\rangle\left\langle e_{k}^{(1)} e_{l}^{(2)}\right|
$$

By defining $S_{\mathrm{OEE}}^{\left(n_{\circ}\right)}\left(\rho_{A_{1} A_{2}}\right)$ as follows

$$
S_{\mathrm{OEE}}^{\left(n_{\circ}\right)}\left(\rho_{A_{1} A_{2}}\right)=\frac{1}{1-n_{\circ}}\left(\operatorname{Tr}\left(\rho_{A_{1} A_{2}}^{T_{A_{2}}}\right)^{n_{\circ}}-1\right),
$$

where $n_{\circ}$ is an odd positive integer, the $S_{\mathrm{OEE}}$ is given by [18]

$$
S_{\mathrm{OEE}}\left(\rho_{A_{1} A_{2}}\right)=\lim _{n_{\circ} \rightarrow 1} S_{\mathrm{OEE}}^{\left(n_{\circ}\right)}
$$

Since, $\rho_{A_{1} A_{2}}^{T_{A_{2}}}$ is a Hermitian operator and the partial transposition is not a completely positive map, then $\rho_{A_{1} A_{2}}^{T_{A_{2}}}$, in general, can have negative eigenvalues. Accordingly, the $S_{\mathrm{OEE}}$ can be written as

$$
S_{\mathrm{OEE}}\left(\rho_{A_{1} A_{2}}\right)=-\sum_{\lambda_{i}>0} \lambda_{i} \log \lambda_{i}+\sum_{\lambda_{i}<0}\left|\lambda_{i}\right| \log \left|\lambda_{i}\right|
$$


where $\lambda_{i}$ 's are the eigenvalues of the $\rho_{A_{1} A_{2}}^{T_{A_{2}}}$ matrix. It is worth mentioning that the OEE reduces to the entanglement entropy for pure states. Moreover, if we subtract the von Neumann entropy $S_{\mathrm{EE}}\left(\rho_{A_{1} A_{2}}\right)$ from OEE, it has been suggested that the obtained quantity might be dual to the entanglement wedge cross-section for holographic theories ${ }^{3}$ [18]

$$
E_{W}\left(\rho_{A_{1} A_{2}}\right)=S_{\mathrm{OEE}}\left(\rho_{A_{1} A_{2}}\right)-S_{\mathrm{EE}}\left(\rho_{A_{1} A_{2}}\right) .
$$

For holographic theories this quantity is positive but, in general, it can be negative. ${ }^{4}$ The LN can also be defined along the same lines:

$$
\mathcal{E}_{A_{1} A_{2}} \equiv \log || \rho_{A_{1} A_{2}}^{T_{A_{2}}} \|=\log \operatorname{Tr}\left|\rho_{A_{1} A_{2}}^{T_{A_{2}}}\right|
$$

where the trace norm $\|\mathcal{O}\| \equiv \operatorname{Tr} \sqrt{\mathcal{O}^{\dagger} \mathcal{O}}$. According to the eigenvalues $\lambda_{i}$ of the operator $\rho_{A_{1} A_{2}}^{T_{A_{2}}}$ one has

$$
\mathcal{E}_{A_{1} A_{2}}=-\sum_{\lambda_{i}>0} \lambda_{i}+\sum_{\lambda_{i}<0}\left|\lambda_{i}\right|
$$

It is easy to see that for pure states, the LN reduces to $n=\frac{1}{2}$ Rényi entropy [16]. It is worth mentioning again that $\mathrm{LN}$ is expected to capture only quantum correlations and has been studied previously in several works [32-44]. Interestingly, it is argued that in the limit of long times and large subsystems with a fix ratio, the LN equals half of the Rényi mutual information

$$
\mathcal{E}\left(A_{1}, A_{2}\right)=\frac{I^{(1 / 2)}\left(A_{1}, A_{2}\right)}{2} .
$$

Accordingly, the condition $\mathcal{E}\left(A_{1}, A_{2}\right)=0$ is necessary (but not sufficient) for the absence of mutual entanglement.

One particular fruitful situation for studying thermalization and global quench in the context of holography has been to study these phenomena for the case of thermofield double states (TFD) [45-47]. Using the dictionary of the AdS/CFT duality, the TFD state is proposed to be dual to the eternal AdS black hole [48] and provides a setup to probe various aspects of black holes from a quantum information theoretic perspective. For example, the TFD state can be used to study scrambling and quantum chaos [49-51], dynamics of entanglement entropy [52], quantum computational complexity [53-56], and so on. This state belongs to the product Hilbert space

$$
\mathcal{H}=\mathcal{H}_{\mathrm{L}} \otimes \mathcal{H}_{\mathrm{R}}
$$

By choosing the following Hamiltonian

$$
H_{\mathrm{TFD}}=H_{L} \otimes I_{R}-I_{L} \otimes H_{R}
$$

\footnotetext{
${ }^{3}$ Although, it has been recently argued that there might be counterexamples for this duality; for more details see [30].

${ }^{4}$ Recently, OEE for Lifshitz scalar theories has been studied in [31] where it has been shown that it can be negative.
} 
the time-dependent TFD state is given by

$$
\left|\operatorname{TFD}\left(t_{L}, t_{R}\right)\right\rangle=\frac{1}{\sqrt{Z(\beta)}} \sum_{n} e^{-\beta E_{n} / 2} e^{-i E_{n}\left(t_{L}+t_{R}\right)}\left|E_{n}\right\rangle_{L}\left|E_{n}\right\rangle_{R}
$$

where $\left|E_{n}\right\rangle_{L, R}$ are the energy eigenstates of the left and right theories (for example two CFTs), respectively, with corresponding times $t_{L, R}$. Moreover, tracing out either copy leads to a thermal state at the inverse temperature $\beta$ for the other, $\rho_{\text {th }}=\frac{1}{Z(\beta)} e^{-\beta H_{i}}, i \equiv L, R$ and $Z(\beta)$ denotes the partition function. According to (1.18) and in the spirit of [52], our setup to consider the dynamics of LN and OEE will be in a rather unusual quantum quench scenario [13] in which two decoupled subsystems are entangled via their initial conditions. Moreover, to have a mixed state, we will consider two spatial non-complementary regions. Since the underlying TFD state is a Gaussian state, we can use the covariance matrix approach to calculate LN and OEE. We will observe that their behaviors under the time evolution can be summarized as a linear growth followed by saturation which is similar to the expectations from the quasi-particle picture. Also, we will observe the oscillatory behavior due to the finite size effect as well as a logarithmic contribution in the intermediate regime due to the existence of the zero-mode.

This paper is organized as follows: in section 2, we will briefly review the reconstruction of the TFD state for two harmonic oscillators and then study its time dependency. Then, we will generalize this to a $1+1$ dimensional free real scalar QFT. We will also introduce the covariance matrix formalism and by using this method in section 3 , we will explain how to evaluate the OEE and LN for subsystems involving an equal number of sites on each spatial side of the TFD state. The numerical results are presented in section 4 . In subsections 4.1 and 4.2, the adjacent and disjoint intervals in each side are studied, respectively. In subsection 4.3, we explore the contribution of different quasi-particles. In subsection 4.4, we compare the evolution of logarithmic negativity with mutual and 1/2-Rényi mutual information and in subsection 4.5, some previously proposed inequalities for OEE are checked. In section 5, we will choose a special dividing for subsystems and give an analytical formula for evaluating the zero-mode contribution. In section 6 , we will conclude and discuss our results and future directions. In appendix A, we study the effect of temperature on the dynamics of entanglement for adjacent and disjoint intervals in each side, separately. In appendix B, we consider the effect of mass on the various entanglement measures. Further details for evaluation of zero-mode contribution and logarithmic growth are provided in appendix $\mathrm{C}$.

\section{Covariance matrix for Gaussian TFD state}

As we have mentioned in the introduction, we would like to study the dynamics of OEE and LN. Accordingly, we will focus on the TFD state of a free real scalar QFT. This TFD state is a Gaussian state and therefore we can use the power of the covariance matrix to calculate the OEE and LN. ${ }^{5}$ Since, in general, the OEE and LN are divergent quantities

\footnotetext{
${ }^{5}$ The Covariance matrix approach has been previously used to probe the dynamics of the entanglement entropy of bosonic and fermionic Gaussian states [13, 57-62].
} 
in the continuum, we will regularize the theory by putting it on a lattice. In the normal mode decomposition, the discretized QFT takes the form of $N$ decoupled simple harmonic oscillators. Accordingly, we will first consider the construction of time-independent as well as time-dependent TFD states for two copies of simple harmonic oscillators. Then, the OEE and LN for the TFD state of discretized QFT will be constructed by a sum on the contribution of each normal mode (simple harmonic oscillator).

\subsection{Free real scalar QFT on a lattice}

The Hamiltonian of a $1+1$ dimensional free real scalar QFT on a circle with a circumference $\mathcal{L}$ is given by

$$
H=\frac{1}{2} \int_{-\frac{\mathcal{L}}{2}}^{\frac{\mathcal{L}}{2}} d x\left(\Pi^{2}+\left(\partial_{x} \Phi\right)^{2}+m^{2} \Phi^{2}\right)
$$

where $\Pi=\frac{\partial L}{\partial \dot{\Phi}}=\dot{\Phi}$ is the conjugate momentum. The regularized Hamiltonian on a circular lattice with $N$ sites and lattice spacing $\delta=\frac{\mathcal{L}}{N}$ becomes

$$
H=\sum_{a=1}^{N}\left(\frac{\delta}{2} \mathbf{P}_{a}^{2}+\frac{m^{2}}{2 \delta} \mathbf{Q}_{a}^{2}+\frac{1}{2 \delta^{3}}\left(\mathbf{Q}_{a+1}-\mathbf{Q}_{a}\right)^{2}\right),
$$

where redefined canonical variables are

$$
\mathbf{Q}_{a}=\Phi\left(x_{a}\right) \delta, \quad \mathbf{P}_{a}=\Pi\left(x_{a}\right),
$$

and we have imposed the periodic boundary conditions $\mathbf{Q}_{N+1}=\mathbf{Q}_{1}$ and $\mathbf{P}_{N+1}=\mathbf{P}_{1}$. By applying the discrete Fourier transformation ${ }^{6}$

$$
\tilde{\mathbf{Q}}_{k}=\frac{1}{\sqrt{N}} \sum_{a=1}^{N} e^{\frac{2 \pi i k a}{N}} \mathbf{Q}_{a}, \quad \tilde{\mathbf{P}}_{k}=\frac{1}{\sqrt{N}} \sum_{a=1}^{N} e^{-\frac{2 \pi i k a}{N}} \mathbf{P}_{a}
$$

where $\tilde{\mathbf{Q}}_{k}^{\dagger}=\tilde{\mathbf{Q}}_{N-k}$ and $\tilde{\mathbf{P}}_{k}^{\dagger}=\tilde{\mathbf{P}}_{N-k}$, the Hamiltonian (2.2) reduces to

$$
H=\sum_{k=0}^{N-1}\left(\frac{\delta}{2}\left|\tilde{\mathbf{P}}_{k}\right|^{2}+\frac{1}{2 \delta} \omega_{k}^{2}\left|\tilde{\mathbf{Q}}_{k}\right|^{2}\right) .
$$

In the above formula, the frequencies $\omega_{k}$ are given by

$$
\omega_{k}^{2}=m^{2}+\frac{4}{\delta^{2}} \sin ^{2}\left(\frac{\pi k}{N}\right) .
$$

To construct the corresponding TFD state, one first needs to quantize the Hamiltonian (2.5) by defining two sets of creation and annihilation operators

$$
\tilde{\mathbf{Q}}_{k}=\sqrt{\frac{\delta}{2 \omega_{k}}}\left(\hat{a}_{N-k}+\hat{a}_{k}^{\dagger}\right), \quad \tilde{\mathbf{P}}_{k}=i \sqrt{\frac{\omega_{k}}{2 \delta}}\left(\hat{a}_{k}^{\dagger}-\hat{a}_{N-k}\right)
$$

\footnotetext{
${ }^{6}$ The canonical commutation relations are given by $\left[\tilde{Q}_{k}, \tilde{P}_{l}^{\dagger}\right]=i \delta_{k, l}$.
} 
where $\left[\hat{a}_{k}, \hat{a}_{k}^{\dagger}\right]=1$. By substituting (2.7) in (2.5) one gets

$$
\hat{H}=\sum_{k=0}^{N-1} \omega_{k}\left(\hat{a}_{k}^{\dagger} \hat{a}_{k}+\frac{1}{2}\right) .
$$

This is the Hamiltonian of $N$ decoupled simple harmonic oscillators with equal mass $M=\delta^{-1}$ (not to be confused with the physical mass $m$ ) and $\mathrm{k}$-dependent frequencies $\omega_{k}$. Ignoring the constant term, the $\hat{a}_{k}^{\dagger} \hat{a}_{k} \omega_{k} \equiv n \omega_{k}$ denotes the total energy of level " $n$ " for each of these oscillators with fixed momentum. Since the zero-mode Hamiltonian does not have a normalizable ground state $\left(\omega_{0}\right.$ vanishes when $\left.m=0\right)$ one can regularize it by introducing a very small dimensionless mass, $m \mathcal{L} \ll 1$. Considering the decoupled form of the Hamiltonian (2.5), the corresponding TFD state of the free scalar theory will be a product of TFD states for each of the oscillator modes. Accordingly, in the following we will focus on a single mode (fixed momentum $k$ ) and construct its time-dependent TFD state.

\subsection{TFD state for a simple harmonic oscillator}

In this subsection, we will first construct the TFD state for a simple harmonic oscillator with mass $m$ and frequency $\omega$ at $t=0$ and then will turn to study its time evolution. The creation and annihilation operators for a simple harmonic oscillator are given by

$$
\hat{a}^{\dagger}=\sqrt{\frac{m \omega}{2}}\left(\hat{Q}-i \frac{\hat{P}}{m \omega}\right), \quad \hat{a}=\sqrt{\frac{m \omega}{2}}\left(\hat{Q}+i \frac{\hat{P}}{m \omega}\right) .
$$

The $n^{t h}$ energy eigenstate is then defined by acting $n$ times with the creation operator on the vacuum state $|0\rangle$ :

$$
|n\rangle=\frac{1}{\sqrt{n !}}\left(\hat{a}^{\dagger}\right)^{n}|0\rangle .
$$

The action of creation and annihilation operators on this state are given by

$$
\hat{a}^{\dagger}|n\rangle=\sqrt{n+1}|n+1\rangle, \quad \hat{a}|n\rangle=\sqrt{n}|n-1\rangle .
$$

The TFD state at $t=0$ can be constructed as a superposition of a tensor product of two copies of the energy eigenstate (2.10), which we label by $L$ and $R$, with special wights [63]:

$$
|\mathrm{TFD}\rangle=Z(\beta)^{-\frac{1}{2}} \sum_{n=0}^{\infty} e^{-\frac{\beta}{2} E_{n}}|n\rangle_{L} \otimes|n\rangle_{R}
$$

where the normalization factor is $Z(\beta)=\left(1-e^{-\beta \omega}\right)^{-1}$ and $E_{n}$ denotes the energy of those eigenstates. Considering this normalization factor together with (2.10), then the state (2.12) can be alternatively written as

$$
|\mathrm{TFD}\rangle=\sqrt{1-e^{-\beta \omega}} \exp \left(e^{-\frac{\beta}{2} \omega} \hat{a}_{L}^{\dagger} \hat{a}_{R}^{\dagger}\right)|0\rangle_{L} \otimes|0\rangle_{R} .
$$

It is worth noting that in (2.13) the operator acting on the total vacuum state is not a unitary operator. It is convenient to re-express (2.13) by acting as a unitary operator on the vacuum state $|0\rangle_{L} \otimes|0\rangle_{R}$. The result is [64]

$$
|\mathrm{TFD}\rangle=e^{\alpha\left(\hat{a}_{L}^{\dagger} \hat{a}_{R}^{\dagger}-\hat{a}_{L} \hat{a}_{R}\right)}|0\rangle_{L} \otimes|0\rangle_{R},
$$


with

$$
\tanh \alpha=e^{-\frac{\beta}{2} \omega}
$$

The time evolution of the state (2.14) is given by

$$
|\operatorname{TFD}(t)\rangle=e^{-i \hat{H}_{L} t_{L}} e^{-i \hat{H}_{R} t_{R}}|\mathrm{TFD}\rangle,
$$

in which the operators $\hat{H}_{L}$ and $\hat{H}_{R}$ are the corresponding Hamiltonians for the left and right simple harmonic oscillators. These quantities are defined as

$$
\hat{H}_{L}=\hat{a}_{L}^{\dagger} \hat{a}_{L}+\frac{1}{2}, \quad \hat{H}_{R}=\hat{a}_{R}^{\dagger} \hat{a}_{R}+\frac{1}{2} .
$$

By choosing $t_{L}=t_{R}=t / 2$, which is the common convention in holography [65], the time dependent TFD state (2.16) takes the following form

$$
|\operatorname{TFD}(t)\rangle=e^{-\frac{i}{2} \omega t} \sqrt{1-e^{-\beta \omega}} \exp \left[e^{-\frac{\beta}{2} \omega} e^{-i \omega t} a_{L}^{\dagger} a_{R}^{\dagger}\right]|0\rangle_{L} \otimes|0\rangle_{R}
$$

One can write the state (2.18) in a more compact form by acting as a unitary operator on the vacuum state $|0\rangle_{L} \otimes|0\rangle_{R}$ as follows $[13,64]^{7}$

$$
|\operatorname{TFD}(t)\rangle=\exp \left[z \hat{a}_{L}^{\dagger} \hat{a}_{R}^{\dagger}-z^{*} \hat{a}_{L} \hat{a}_{R}\right]|0\rangle_{L} \otimes|0\rangle_{R}
$$

where

$$
z=\alpha e^{-i \omega t}
$$

and $\alpha$ is the same as (2.15). Now by having the time-dependent TFD state (2.19), one needs to find the covariance matrix associated with it. That is the subject of the next subsection.

\subsection{Covariance matrix formalism}

The system (2.5) is described by $2 N$ linear observables $\hat{\xi}=\left(\hat{q}_{1}, \hat{q}_{2}, \ldots, \hat{q}_{N}, \hat{p}_{1}, \ldots, \hat{p}_{N}\right)$. The $\left(\hat{q}_{i}, \hat{p}_{i}\right)$ are canonical operators where $\left[\hat{q}_{i}, \hat{p}_{j}\right]=i \delta_{i j}$. The two-point functions of these observables in an arbitrary state $|\Psi\rangle$ can be decomposed as

$$
\left\langle\Psi\left|\hat{\xi}^{a} \hat{\xi}^{b}\right| \Psi\right\rangle=\frac{1}{2}\left(G^{a b}+i \Omega^{a b}\right)
$$

where $G^{a b}=G^{(a b)}$ and $\Omega^{a b}=\Omega^{[a b]}$ are the symmetric and the antisymmetric parts of the correlation functions, respectively. For a bosonic state, $\Omega^{a b}$ is completely fixed by the commutation relations of $\hat{q}_{i}$ and $\hat{p}_{i}$,

$$
\Omega^{a b}=\left(\begin{array}{cc}
0 & 1 \\
-1 & 0
\end{array}\right)
$$

\footnotetext{
${ }^{7}$ Here, we have dropped the global time-dependent phase, since this does not change the physical state.
} 
For a pure Gaussian state with vanishing first moment $\left\langle\psi\left|\hat{\xi}^{a}\right| \psi\right\rangle=0$, the covariance matrix is given by the symmetric part of the two-point function

$$
G^{a b}=\left\langle\Psi\left|\hat{\xi}^{a} \hat{\xi}^{b}+\hat{\xi}^{b} \hat{\xi}^{a}\right| \Psi\right\rangle
$$

By using Wick's theorem, one can compute all of the $n$-point functions from $G^{a b}$. Hence, it can be used to label the Gaussian states. For a mixed state $\rho$, when $\left\langle\Psi\left|\hat{\xi}^{a}\right| \Psi\right\rangle=0$, the covariance matrix is defined by $[66,67]$

$$
G^{a b}=\operatorname{Tr}\left(\rho\left(\hat{\xi}^{a} \hat{\xi}^{b}+\hat{\xi}^{b} \hat{\xi}^{a}\right)\right) .
$$

To find the covariance matrix associated with the TFD state (2.14), one can first find the covariance matrix for the vacuum state and then by a unitary transformation modify it for the TFD state. To do so, we restrict ourselves to the space of Gaussian states. In this space, the general unitary operator $\hat{U}(s)$ can be expressed by Hermitian operators which are quadratic in the canonical operators $\hat{\xi}$,

$$
\hat{U}(s)=e^{-i s \hat{K}}, \quad \text { with } \quad \hat{K}=\frac{1}{2} \hat{\xi}^{a} k_{(a, b)} \hat{\xi}^{b} \equiv \frac{1}{2} \hat{\xi} k \hat{\xi}^{T} .
$$

Accordingly, the transformed state is

$$
\left|G_{s}\right\rangle=\hat{U}(s)\left|G_{0}\right\rangle,
$$

where the subscript "0" indicates the vacuum state (for the time-dependent state (2.19) it refers to the TFD state (2.14)). To find the corresponding covariance matrix, one needs the operation of $\hat{U}(s)$ on $\hat{\xi}^{a}$ which can be obtained as follows

$$
\hat{U}^{\dagger}(s) \hat{\xi}^{a} \hat{U}(s)=\sum_{n=0}^{\infty} \frac{s^{n}}{n !}\left[i \hat{K}, \hat{\xi}^{a}\right]_{(n)} .
$$

In the above formula, $\left[i \hat{K}, \hat{\xi}^{a}\right]_{(n)}$ is defined recursively by $\left[i \hat{K}, \hat{\xi}^{a}\right]_{(n)}=\left[i \hat{K},\left[i \hat{K}, \hat{\xi}^{a}\right]_{(n-1)}\right]$, and $\left[i \hat{K}, \hat{\xi}^{a}\right]_{(0)}=\left[i \hat{K}, \hat{\xi}^{a}\right]$. With respect to the $(2.25)$ and the commutation relation $\left[\hat{\xi}^{a}, \hat{\xi}^{b}\right]=i \Omega^{a b}$, one can find that

$$
\left[i \hat{K}, \hat{\xi}^{a}\right]=\Omega^{a b} k_{(b, c)} \hat{\xi}^{c} .
$$

By defining $K_{b}^{a}=\Omega^{a c} k_{(c, b)}$, the above formula can be rewritten as

$$
\left[i \hat{K}, \hat{\xi}^{a}\right]=K_{b}^{a} \hat{\xi}^{b}
$$

Hence, the operation of $\hat{U}(s)$ on $\hat{\xi}^{a},(2.27)$, can be expressed as follows

$$
\hat{U}^{\dagger}(s) \hat{\xi}^{a} \hat{U}(s)=\left(e^{s K}\right)_{b}^{a} \hat{\xi}^{b} \equiv U(s)_{b}^{a} \hat{\xi}^{b} .
$$

Now, the relations (2.25) together with the relation (2.30) implies that the covariance matrix associated with transformed state $\left|G_{s}\right\rangle$ becomes

$$
G_{s}^{(a, b)}=\left\langle G_{s}\left|\left(\hat{\xi}^{a} \hat{\xi}^{b}+\hat{\xi}^{b} \hat{\xi}^{a}\right)\right| G_{s}\right\rangle=U(s)_{c}^{a} G_{0}^{(c, d)} U(s)_{d}^{b},
$$


where $G_{0}^{(c, d)}$ is its counterpart for the vacuum. Accordingly, in the compact notation, we have

$$
\left|G_{s}\right\rangle=\hat{U}(s)\left|G_{0}\right\rangle=\left|U(s) G_{0} U^{\top}(s)\right\rangle, \quad G_{s}=U(s) G_{0} U^{\top}(s) .
$$

In the following, we will demonstrate how to evaluate the covariance matrix associated with the time-independent as well as time-dependent TFD states. This is easily achieved by using the above formalism.

\subsubsection{Covariance matrix for TFD state of the harmonic oscillator}

Let us begin with constructing the covariance matrix associated with the time-independent TFD state (2.14) corresponding to $N=1$ and then extend it to the time-dependent case (2.19). The Hamiltonian of the system is described by

$$
\hat{H}=\frac{1}{2 m}\left(\hat{p}_{L}^{2}+\hat{p}_{R}^{2}+m^{2} \omega^{2}\left(\hat{q}_{L}^{2}+\hat{q}_{R}^{2}\right)\right) .
$$

By rewritting (2.14) in terms of the $\left(q_{L}, p_{L}, q_{R}, p_{R}\right)$ coordinates and reading the corresponding $\hat{K}$ and $U(s)$, the covariance matrix of TFD state (2.14) for a single-mode is given by

$$
G_{\alpha}^{a b}=\left(\begin{array}{cccc}
\frac{\cosh (2 \alpha)}{m \omega} & 0 & -\frac{\sinh (2 \alpha)}{m \omega} & 0 \\
0 & m \omega \cosh (2 \alpha) & 0 & m \omega \sinh (2 \alpha) \\
-\frac{\sinh (2 \alpha)}{m \omega} & 0 & \frac{\cosh (2 \alpha)}{m \omega} & 0 \\
0 & m \omega \sinh (2 \alpha) & 0 & m \omega \cosh (2 \alpha)
\end{array}\right) .
$$

The time-dependent covariance matrix associated with time-dependent TFD state (2.19) can be derived as follows. The Hamiltonian (2.33) has the form $\hat{H}=\frac{1}{2} \hat{\xi^{a}} k_{a b} \hat{\xi^{b}}$, where the matrix representation of $k_{a b}$ with respect to $\hat{\xi}=\left(\hat{q}_{L}, \hat{p}_{L}, \hat{q}_{R}, \hat{p}_{R}\right)$ is given by

$$
k_{a b}=\left(\begin{array}{cccc}
m \omega^{2} & 0 & 0 & 0 \\
0 & \frac{1}{m} & 0 & 0 \\
0 & 0 & m \omega^{2} & 0 \\
0 & 0 & 0 & \frac{1}{m}
\end{array}\right) .
$$

The simplectic generator $K_{b}^{a}=\Omega^{a c} k_{(c, b)}$ becomes

$$
K_{b}^{a}=\left(\begin{array}{cccc}
0 & \frac{1}{m} & 0 & 0 \\
-m \omega^{2} & 0 & 0 & 0 \\
0 & 0 & 0 & \frac{1}{m} \\
0 & 0 & -m \omega^{2} & 0
\end{array}\right),
$$

and therefore

$$
U(t)=\exp (t K)=\left(\begin{array}{cccc}
m \cos (\omega t) & \frac{\sin (\omega t)}{m} & 0 & 0 \\
-\frac{\sin (\omega t)}{m} & m \cos (\omega t) & 0 & 0 \\
0 & 0 & m \cos (\omega t) & \frac{\sin (\omega t)}{m} \\
0 & 0 & -\frac{\sin (\omega t)}{m} & m \cos (\omega t)
\end{array}\right) .
$$


Using this and noting to (2.34), the covariance matrix $G_{\alpha}^{a b}(t)=U(t) G_{\alpha}^{a b} U^{\top}(t)$ becomes

$$
G_{\alpha}^{a b}(t)=\left(\begin{array}{cccc}
\frac{\cosh (2 \alpha)}{m \omega} & 0 & -\frac{\sinh (2 \alpha) \cos (\omega t)}{m \omega} & \sin (\omega t) \sinh (2 \alpha) \\
0 & m \omega \cosh (2 \alpha) & \sin (\omega t) \sinh (2 \alpha) & m \omega \sinh (2 \alpha) \cos (\omega t) \\
-\frac{\sinh (2 \alpha) \cos (\omega t)}{m \omega} & \sin (\omega t) \sinh (2 \alpha) & \frac{\cosh (2 \alpha)}{m \omega} & 0 \\
\sin (\omega t) \sinh (2 \alpha) & m \omega \sinh (2 \alpha) \cos (\omega t) & 0 & m \omega \cosh (2 \alpha)
\end{array}\right) .
$$

\subsubsection{Covariance matrix for TFD state of real scalar QFT}

As we explained previously, according to the discretized Hamiltonian (2.5) on a lattice with $N$ sites, the TFD state (2.19) is described by $2 N$ degrees of freedom on each side. Again, one can choose the following coordinates

$$
\xi^{a}=\left(q_{m, L}, p_{m, L}, q_{m, R}, p_{m, R}\right),
$$

where $q_{m}=\frac{1}{\sqrt{N}} \Phi_{L}\left(x_{m}\right), p_{m}=\frac{\mathcal{L}}{\sqrt{N}} \Pi_{L}\left(x_{m}\right)$, and $x_{m}=m \delta$ which $\delta$ is the distance between the two sites. Using the Fourier space coordinates

$$
\tilde{q}_{k}=\frac{1}{\sqrt{N}} \sum_{m=1}^{N} e^{\frac{2 \pi i k m}{N}} q_{m}, \quad \tilde{p}_{k}=\frac{1}{\sqrt{N}} \sum_{m=1}^{N} e^{-\frac{2 \pi i k m}{N}} p_{m},
$$

and the extension of (2.38), the covariance matrix associated with the time-dependent TFD state for a real scalar QFT (on the lattice) is real $[13,62,68]$ and is given by

$$
G(t)=\left(\begin{array}{cccc}
G_{L L}^{\Phi \Phi} & G_{L L}^{\Phi \Pi} & G_{L R}^{\Phi \Phi} & G_{L R}^{\Phi \Pi} \\
G_{L L}^{\Pi \Phi} & G_{L L}^{\Pi \Pi} & G_{L R}^{\Pi \Phi} & G_{L R}^{\Pi \Pi} \\
G_{R L}^{\Phi \Phi} & G_{R L}^{\Phi \Pi} & G_{R R}^{\Phi \Phi} & G_{R R}^{\Phi \Pi} \\
G_{R L}^{\Pi \Phi} & G_{R L}^{\Pi \Pi} & G_{R R}^{\Pi \Phi} & G_{R R}^{\Pi \Pi}
\end{array}\right)
$$

where,

$$
\begin{aligned}
& G_{L L}^{\Phi \Phi}=G_{R R}^{\Phi \Phi}=\frac{1}{N} \sum_{k=0}^{N-1} e^{\frac{2 \pi i k(m-n)}{N}} \frac{\cosh \left(2 \alpha_{k}\right)}{\lambda_{k}} \\
& G_{L L}^{\Pi \Pi}=G_{R R}^{\Pi \Pi}=\frac{1}{N} \sum_{k=0}^{N-1} e^{\frac{2 \pi i k(m-n)}{N}} \lambda_{k} \cosh \left(2 \alpha_{k}\right), \\
& G_{L L}^{\Phi \Pi}=G_{L L}^{\Pi \Phi}=G_{R R}^{\Phi \Pi}=G_{R R}^{\Pi \Phi}=0, \\
& G_{L R}^{\Phi \Phi}=G_{R L}^{\Phi \Phi}=-\frac{1}{N} \sum_{k=0}^{N-1} e^{\frac{2 \pi i k(m-n)}{N}} \frac{\cos \left(\omega_{k} t\right) \sinh \left(2 \alpha_{k}\right)}{\lambda_{k}} \\
& G_{L R}^{\Pi \Pi}=G_{R L}^{\Pi \Pi}=\frac{1}{N} \sum_{k=0}^{N-1} e^{\frac{2 \pi i k(m-n)}{N}} \lambda_{k} \cos \left(\omega_{k} t\right) \sinh \left(2 \alpha_{k}\right), \\
& G_{L R}^{\Phi \Pi}=G_{L R}^{\Pi \Phi}=G_{R L}^{\Phi \Pi}=G_{R L}^{\Pi \Phi}=\frac{1}{N} \sum_{k=0}^{N-1} e^{\frac{2 \pi i k(m-n)}{N}} \sin \left(\omega_{k} t\right) \sinh \left(2 \alpha_{k}\right),
\end{aligned}
$$


with $\alpha_{k}=\frac{1}{2} \log \operatorname{coth}\left(\frac{\beta \omega_{k}}{4}\right), \lambda_{k}=\mathcal{L} \omega_{k}$ and $\mathcal{L}$ denote the circumference of a circle. Note that $L$ or $R$ are separately described by a thermal state so the associated blocks are timeindependent. But the crossing blocks, $L R$ and $R L$, correspond to correlations between the left and right sides and are time-dependent. In the next section, we will see that the time dependence of OEE and LN comes from these blocks.

\section{Time evolution of $S_{\mathrm{OEE}}$ and $\mathcal{E}$}

In this section, using the mentioned covariance matrix formalism, we will investigate the time evolution of OEE and LN for the time-dependent $1+1$ TFD state of a real scalar QFT regularized on a circular lattice. In above it is shown that this state is just a product of TFD states for each of the oscillator modes, (2.19). In order to evaluate these quantum information quantities we will take a subregion of the entire quantum system that contains a part in both the left and the right QFTs, see figure 1. Let us describe the whole system as

$$
\left(A_{L} \cup B_{L}\right) \cup\left(A_{R} \cup B_{R}\right),
$$

in the corresponding TFD state. According to figure 1, the $B_{L(R)}$ are the complement of $A_{L(R)}$. Moreover, we decompose the region $A_{L(R)}$ into two subregions $A_{L_{1}\left(R_{1}\right)}$ and $A_{L_{2}\left(R_{2}\right)}$ where they describe two (in general disconnected) subregions on each side

$$
\left(\left(A_{L_{1}} \cup A_{L_{2}}\right) \cup B_{L}\right) \cup\left(\left(A_{R_{1}} \cup A_{R_{2}}\right) \cup B_{R}\right) .
$$

We would like to study $S_{\mathrm{OEE}}\left(\rho_{12}(t)\right)$ and $\mathcal{E}\left(\rho_{12}(t)\right)$ between two non-complementary regions $A_{1}=\left(A_{L_{1}} \cup A_{R_{1}}\right)$ and $A_{2}=\left(A_{L_{2}} \cup A_{R_{2}}\right)$. The reduced covariance matrix $G_{12}$ can be obtained from the covariance matrix $(2.41)$ where each of the regions $\left(A_{L_{1}} \cup A_{L_{2}}\right)$ and $\left(A_{R_{1}} \cup\right.$ $A_{R_{2}}$ ) contain $N_{A}$ sites. Accordingly, $G_{12}$ is decomposed into four blocks corresponding to $L R$ decomposition in position space such that each block is a $2 N_{A} \times 2 N_{A}$ matrix. Two of these blocks, $G_{12}^{L L}$ and $G_{12}^{R R}$, are time-independent and the time dependence of the covariance matrix comes from mixed blocks, $G_{12}^{L R}(t)$ and $G_{12}^{R L}(t)$ :

$$
G_{12}^{a b}(t)=\left(\begin{array}{cc}
G_{12}^{L L} & G_{12}^{L R}(t) \\
G_{12}^{R L}(t) & G_{12}^{R R}
\end{array}\right)_{4 N_{A} \times 4 N_{A}}
$$

with

$$
G_{12}^{L L}=\left(\begin{array}{cc}
G_{L L}^{\Phi \Phi} & 0 \\
0 & G_{L L}^{\Pi \Pi}
\end{array}\right)_{2 N_{A} \times 2 N_{A}} \quad G_{12}^{L R}(t)=\left(\begin{array}{c}
G_{L R}^{\Phi \Phi} G_{L R}^{\Phi \Pi} \\
G_{L R}^{\Pi \Phi} G_{L R}^{\Pi \Pi}
\end{array}\right)_{2 N_{A} \times 2 N_{A}}
$$

In the above expression, $\left\{G_{L L}^{\Phi \Phi}, G_{L L}^{\Pi \Pi}, \ldots\right\}$ are given by the equation $(2.42)$ with $m, n$ restricted to the entangling region. In addition, $G_{12}^{R R}, G_{12}^{R L}(t)$ blocks can be obtained by changing $L$ by $R$ in (3.4). Once the covariance matrix $G_{12}^{a b}(t),(3.3)$, is found one can express the $S_{\mathrm{EE}}\left(\rho_{12}(t)\right)$ in terms of eigenvalues $\left(\nu_{i}\right)$ of the symplectic form

$$
J=i \Omega_{A}^{-1} G_{12}(t) .
$$



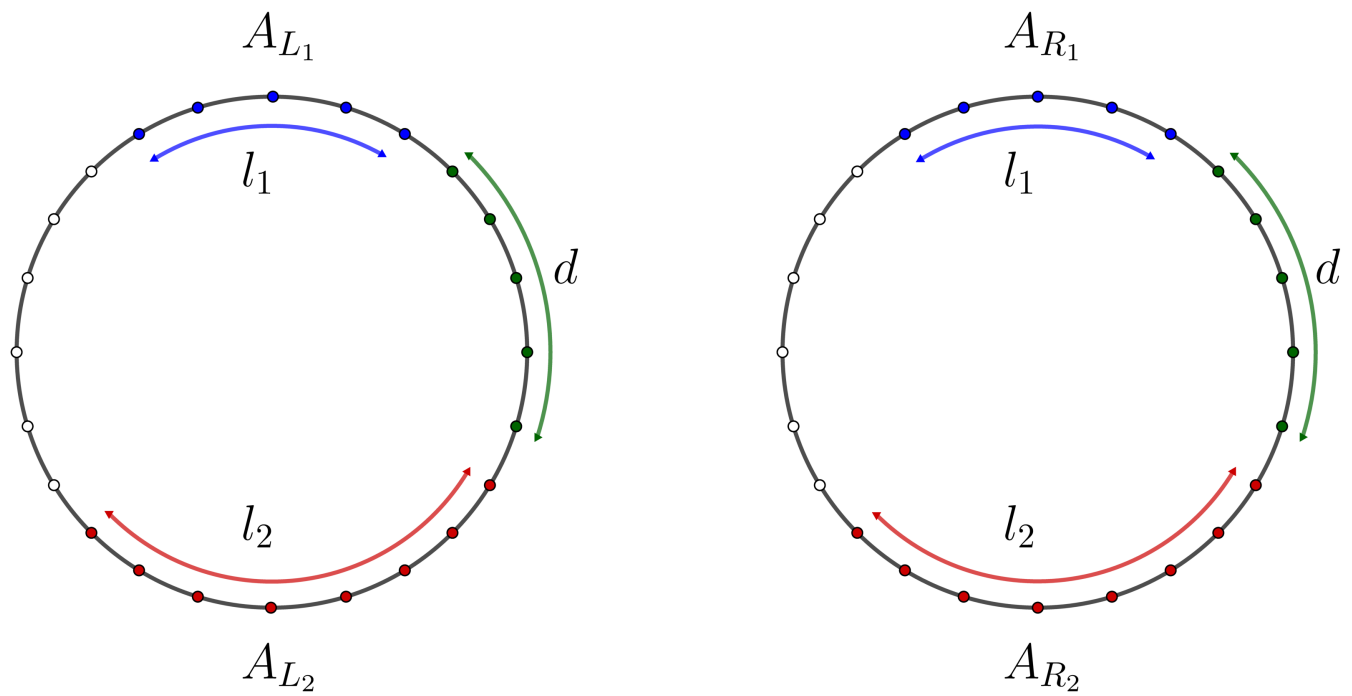

Figure 1. Our setup for the decomposition of the system is depicted in this figure. We have taken $N$ sites on each side and the total subsystem consists of four spatially disconnected regions: two intervals on the left $\left(A_{L}\right)$ with the separation $d$ and the corresponding (identical) intervals and the same separation on the right $\left(A_{R}\right)$ with $N_{A}$ sites on each side.

Doing so, one obtains $[66,67]:^{8}$

$$
S_{\mathrm{EE}}\left(\rho_{12}(t)\right)=\frac{1}{2} \sum_{i=1}^{4 N_{A}} s_{\mathrm{EE}}\left(\left|\nu_{i}\right|\right),
$$

where,

$$
s_{\mathrm{EE}}\left(\nu_{i}\right)=\left(\frac{\nu_{i}+1}{2}\right) \log \left(\frac{\nu_{i}+1}{2}\right)-\left(\frac{\nu_{i}-1}{2}\right) \log \left(\frac{\nu_{i}-1}{2}\right) .
$$

It is useful to mention that the Rényi entropies $S_{n}$ for $n>0$ can be computed by replacing the $s_{\mathrm{EE}}\left(\nu_{i}\right)$ in $(3.6)$ with $s_{n}\left(\nu_{i}\right)$ defined as

$$
s_{n}\left(\nu_{i}\right)=\frac{1}{n-1} \log \left[\frac{\left(\nu_{i}+1\right)^{n}-\left(\nu_{i}-1\right)^{n}}{2^{n}}\right] .
$$

The entanglement entropy (3.6) can be recovered in the limit $n \rightarrow 1$. In addition, the second Rényi entropy is $S_{2}=\frac{1}{2} \log \operatorname{det}(G)$. Now, in order to compute $S_{\mathrm{OEE}}$, we must take a partial transpose with respect to momentum degrees of freedom in the $A_{2}$ subregion [34,35]. This can be accomplished by acting with the time-reversal operator $\mathcal{R}_{A_{2}}$ on each block of $G_{12}^{a b}$. For example

$$
\tilde{G}_{12}^{L L}=\mathcal{R}_{A_{2}} \cdot G_{12}^{L L} \cdot \mathcal{R}_{A_{2}},
$$

with $\mathcal{R}_{A_{2}}$ given by a $2 N_{A} \times 2 N_{A}$ square matrix:

$$
\mathcal{R}_{A_{2}}=\operatorname{diag}\{1,1, \cdots, 1,-1, \cdots,-1\}
$$

\footnotetext{
${ }^{8}$ We are taking the absolute value of the eigenvalues of the symplectic form. Since these eigenvalues come in pairs, we should include a factor of $\frac{1}{2}$ in evaluating the entanglement entropy.
} 
The number of "-1" elements in the above expression is equal to the length of the subregion $A_{L_{2}}$. It is worth mentioning that the operator $\mathcal{R}_{A_{2}}$ is the same for the other blocks, $G_{12}^{L R}(t), G_{12}^{R L}(t)$ and $G_{12}^{R R}(t)$. This is because we are considering the simplest case here in which the $L$ and $R$ blocks are exactly the same. Now, by having $\tilde{G}_{12}^{a b}$, one can compute eigenvalues $\left(\tilde{\nu}_{i}\right)$ of partial transposed symplectic form

$$
\tilde{J}=i \Omega_{A}^{-1} \tilde{G}_{A_{1} A_{2}}
$$

Having these, the odd entanglement entropy, $S_{\mathrm{OEE}}$, becomes [35]

$$
S_{\mathrm{OEE}}\left(\rho_{12}(t)\right)=\frac{1}{2} \sum_{i=1}^{4 N_{A}} \tilde{s}_{\text {odd }}\left(\left|\tilde{\nu}_{i}\right|\right)
$$

with

$$
\tilde{s}_{\text {odd }}\left(\tilde{\nu}_{i}\right)=\left(\frac{\tilde{\nu}_{i}+1}{2}\right) \log \left(\frac{\tilde{\nu}_{i}+1}{2}\right)-\operatorname{sgn}\left(\frac{\tilde{\nu}_{i}-1}{2}\right)\left|\frac{\tilde{\nu}_{i}-1}{2}\right| \log \left(\left|\frac{\tilde{\nu}_{i}-1}{2}\right|\right) .
$$

It is worth emphasizing that the partial transposition is not a completely positive map and the appearance of negative eigenvalues is a sign of quantum entanglement [32]. This is the main reason to consider the absolute value in (3.13) in comparison with (3.7). Moreover, by knowing the eigenvalues $\tilde{\nu_{i}}$, the trace norm of the reduced density matrix $\rho_{12}^{T_{2}}$ becomes $[35,37]^{9}$

$$
\operatorname{Tr}\left|\rho_{12}^{T_{2}}\right|=\prod_{i=1}^{4 N_{A}}\left[\left|\frac{\tilde{\nu}_{i}+1}{2}\right|-\left|\frac{\tilde{\nu}_{i}-1}{2}\right|\right]^{-1}=\prod_{i=1}^{4 N_{A}} \max \left(1, \frac{1}{\tilde{\nu}_{i}}\right),
$$

which implies that the LN becomes ${ }^{10}$

$$
\mathcal{E}\left(A_{1}, A_{2}\right)=-\frac{1}{2} \sum_{i=1}^{4 N_{A}} \log \left[\min \left(1, \tilde{\nu}_{i}\right)\right] .
$$

According to the above relation, only the symplectic eigenvalues $\tilde{\nu}<1$ contribute to the LN. Hence, in order to have quantum correlation we must have at least one symplectic eigenvalue which is less than one. Note that the LN is a relative entanglement measure, hence, it is symmetric with respect to the exchange of subsystems.

Before closing this section, let us clarify some points that will be useful for interpreting the numerical results presented in the next section. The entanglement entropy enjoys several properties. One of them is known as subadditivity. With respect to the decomposition

$$
A=\left(A_{L_{1}} \cup A_{L_{2}}\right) \cup\left(A_{R_{1}} \cup A_{R_{2}}\right),
$$

the entanglement entropy satisfies

$$
S_{\mathrm{EE}}\left(\rho_{A}(t)\right) \leqslant S_{\mathrm{EE}}\left(\rho_{A_{L}}(t)\right)+S_{\mathrm{EE}}\left(\rho_{A_{R}}(t)\right),
$$

\footnotetext{
${ }^{9}$ The out-of-equilibrium dynamics of the negativity after a different quench has been studied previously in several works $[28,29,44,69-73]$.

${ }^{10}$ The factor $1 / 2$ comes from the fact that we are taking the absolute values of eigenvalues which come in pairs.
} 
in which, $A_{L(R)}=A_{L_{1}\left(R_{1}\right)} \cup A_{L_{2}\left(R_{2}\right)}$. The subadditivity provides a time-independent upper bound for the entanglement entropy. The aforementioned decomposition results in a block structure in the reduced covariance matrix $G_{A}$ which consists of four blocks corresponding to $L R$ decomposition in the position space. The time-independent blocks, $G_{12}^{L L}$ and $G_{12}^{R R}$, are related to thermal entropy corresponding to reduced thermal density matrix of each individual side, $\left(A_{L_{1}} \cup A_{L_{2}}\right)$ and $\left(A_{R_{1}} \cup A_{R_{2}}\right)$. Since intervals are equal and symmetric, these thermal entropies are equal. Therefore, the time-independent upper bound is twice the thermal entropy. This means that with respect to the bound (3.17), the growing behavior of the entanglement entropy over a large range of times ultimately terminates in twice the thermal entropy.

There is also another inequality that is specific to the Gaussian states [57],

$$
S_{\mathrm{EE}}\left(\rho_{A}(t)\right) \leqslant S_{2}\left(\rho_{A}(t)\right)+2 N_{A}(1-\log 2) .
$$

In the above inequality, $N_{A}$ denotes the number of bosonic degrees of freedom associated with each side of the TFD state in the subregion $A$ and $S_{2}\left(\rho_{A_{L}}(t)\right)=\frac{1}{2} \log \operatorname{det} G_{A}(t)$. The inequality (3.18) can be derived using the below expressions for entanglement entropy and second Rényi entropy,

$$
S_{\mathrm{EE}}\left(\rho_{A}(t)\right)=\frac{1}{2} \sum_{i=1}^{4 N_{A}} s\left(\nu_{i}\right), \quad S_{2}=\frac{1}{2} \sum_{i=1}^{4 N_{A}} \log \left(\nu_{i}\right),
$$

where $s\left(\nu_{i}\right)$ is given by (3.7). According to (3.7), for all $\nu \geqslant 1$, we have

$$
\log (\nu) \leqslant s(\nu) \leqslant \log (\nu)+(1-\log 2) .
$$

Therefore, by summing over all eigenvalues we can conclude that the second Rényi entropy provides an upper bound for entanglement entropy of Gaussian states. We will especially use the inequality (3.18) in the analysis of zero-mode in section 5 .

\section{Numerical results}

In this section, we will present our numerical results for the time evolution of OEE and LN based on equations (3.12) and (3.15). These results are expressed in terms of two dimensionless parameters $m \mathcal{L}$ and $\beta / \mathcal{L}$ where $m$ refers to the mass parameter in $(2.1), \mathcal{L}$ is the length of the circular lattice with lattice spacing $\delta, \mathcal{L}=N \delta$, and $\beta$ is the inverse temperature. Let us remind that we are decomposing the circular lattice according to (3.2). The subsystem $A$ is further decomposed into two subsystems $A_{1}$ and $A_{2}$ with length $l_{1}$ and $l_{2}$ respectively:

$$
N_{A}=N_{A_{1}}+N_{A_{2}}=\left(l_{1}+l_{2}\right) / \delta=l / \delta .
$$

Since we are interested in describing the TFD state (for single-mode see (2.19)), one has two copies of this decomposition: one for the left QFT and one for the right QFT where on each side we have $N_{A}$ sites (See figure 1). In what follows, we will study different 
cases for massless scalar QFTs. The similar study for the massive case is provided in appendix B. We consider the behavior of OEE and LN by changing $N_{A}\left(N_{A_{1}}\right.$ or $N_{A_{2}}$ or both of them), the separation distance $d$ (between $A_{1}$ and $A_{2}$ on each side), lattice spacing $\delta$ and inverse temperature $\beta$ (appendix A). The obtained results will also be compared with their counterparts for the entanglement entropy. To do so, we will discretize the circular lattice with circumference $\mathcal{L}$ into $N=2 n+1$ sites (almost 1501) and the length of intervals $l_{1}$ and $l_{2}$ on each side vary from $0.1 \mathcal{L}$ to $0.5 \mathcal{L}$. Before going to the details of numerical results, it is worth to explain why we choose the lattice cites an odd number. It is clear from (2.3) that $\mathbf{P}_{a}$ and $\mathbf{Q}_{a}$ are real degrees of freedom., i.e. $\mathbf{P}_{a}^{\dagger}=\mathbf{P}_{a}$ and $\mathbf{Q}_{a}^{\dagger}=\mathbf{Q}_{a}$. But when we pass to the normal mode basis by Fourier transforming, the $\tilde{\mathbf{P}}_{k}$ and $\tilde{\mathbf{Q}}_{k}$ are no longer real with the exceptions of $k=0$ and $k=N / 2$ (for even $N$ ). To restore (each) two real degrees of freedom, we have imposed the constraints $\tilde{\mathbf{P}}_{k}^{\dagger}=\tilde{\mathbf{P}}_{-k}$ and $\tilde{\mathbf{Q}}_{k}^{\dagger}=\tilde{\mathbf{Q}}_{-k}$ which imply that the negative and positive momentum modes will be mixed, $\left[\tilde{\mathbf{Q}}_{k}, \tilde{\mathbf{P}}_{-l}\right]=i \delta_{k, l}$. Accordingly, to avoid over-counting (constraining) those two excepted modes, we choose $N=2 n+1$ for all calculations.

\subsection{Adjacent intervals on each side}

Let us first consider the case in which the two intervals $A_{1}$ and $A_{2}$ on each side are adjacent to each other, i.e., the separation distance vanishes, $d=0$. In figure 2 , we investigate the time dependence of entanglement entropy $S_{\mathrm{EE}}$ (upper plots), odd entanglement entropy $S_{\mathrm{OEE}}$ (middle plots) and logarithmic negativity $\mathcal{E}$ (bottom plots) in massless theory for short-time (left plots) and long-time (right plots) scales where it is subtracted and normalized with respect to the thermal entropy $S_{\mathrm{th}} \cdot{ }^{11}$ For short-times (i.e. times smaller than $\mathcal{L} / 2$ where the system is not sensitive to finite size effects) the growth of $S_{\mathrm{EE}}$ as well as $S_{\mathrm{OEE}}$ is linear and lasts until approximately $t \sim l$; this linear growth is then followed by saturation. For long-times (i.e. times larger than $\mathcal{L}-l$ where the finite size effects can be visible) the time dependency of $S_{\mathrm{EE}}$ (upper-right panel) as well as $S_{\mathrm{OEE}}$ (middle-right panel) is periodic with periodicity $\mathcal{L}$. To be more precise, the pattern of the time evolution of $S_{\mathrm{EE}}$, as well as $S_{\mathrm{OEE}}$, is consisting of linear growth for early times, $t \sim l$, a quasi-plateau of width approximately $\mathcal{L}-2 l$ in the intermediate times and a linear decreasing up to order $t \sim l$ and then repeating the same structure. ${ }^{12}$ The slope of linear growth, for both of these quantities, is equal with good accuracy to two times of thermal entropy density of each copy at inverse temperature $\beta$. This matches with (1.3), since in massless theory the group velocity is the same for all species of quasi-particles and the slope becomes the sum of entropy densities (1.4) over all species. We called it quasi-plateau since for these flat regimes we observe a logarithmic growth. In section 5, we explicitly show that this non-trivial growth is due to the zero momentum mode. It is also worth mentioning that, upon a closer examination of figure 2, the linear regime does not start right away and a different behavior

\footnotetext{
${ }^{11}$ For a real scalar QFT, the thermal entropy in the continuum limit is given by

$$
S_{\mathrm{th}}=\operatorname{vol} \int \frac{\mathrm{d}^{d-1} k}{(2 \pi)^{d-1}}\left[\frac{\beta\left(\omega_{k}\right)}{e^{\beta\left(\omega_{k}\right)}-1}-\log \left(1-e^{-\beta\left(\omega_{k}\right)}\right)\right] .
$$

${ }^{12}$ Similar revivals of quantum states after different quantum quench has been already seen [74-76].
} 
can be seen around $t=0$ which we believe is a manifestation of an expected quadratic growth at early times following a quantum quench. This quadratic growth is universal i.e. it exists not only for free theories but also for strong interacting ones such as holographic CFTs [47]. The time dependence of LN is depicted in the bottom plots. In early times (the bottom-left panel), we observe growth and then decreasing followed by saturation. The oscillatory behavior can be observed for long times (the bottom-right panel) due to the finite size effects. This can also be understood by a quasi-particle picture where meeting quasi-particles on the opposite side of the circle leads to reducing the correlations between the subsystem and its complement. An important feature of $\mathcal{E}$ is the sudden decreasing of entanglement. It is worth mentioning that one should not worry about growing behavior over a large range of times for $S_{\mathrm{EE}}$ since according to (3.17) we know that this growth ultimately terminates. The similar behavior of $S_{\mathrm{OEE}}$ over long times is then the sign that this quantity also has a sub-additivity characteristic.

In figure 3 , we consider the time dependence of $\Delta S=S_{\mathrm{OEE}}-S_{\mathrm{EE}}$ which is normalized with respect to thermodynamic entropy $S_{\text {th }}$ and subtracted from initial value for massless theory. Accordingly, $\Delta S(t)-\Delta S(0)$ initially increases and then decreases followed by saturation. This behavior is same as the time evolution of logarithmic negativity $\mathcal{E}$ (figure 2) and it will be used to explore the interpretation of numerical results in subsection 4.3. In the right panel, the long time behavior is plotted and the finite size effect can be seen.

In figure 4 , we explore the decompactification limit (equivalently continuous limit) when theory lives on a line instead of a circle. ${ }^{13}$ For this purpose, we keep fix the lattice spacing $\delta$, mass $m$, and inverse temperature $\beta$ while increase the total number of lattice sites $N$. We observe a time delay to see the oscillatory behavior for the larger $N$. In another words, finite size effects are pushed to later and later times by increasing the radius of circle on which theory lives. Moreover, we observe that the coefficient of logarithmic growth becomes smaller by increasing the value of $N$. This is consistent with the result of next section since for the fixed mass and fixed lattice spacing and increasing the system size we expect to only see the initial contribution in (5.14) where the coefficient of logarithmic growth changes from 1 to $1 / 2$. The existence of this long-lived logarithmic growth comes back to the non-local nature of the zero-mode and it is related to the periodic boundary condition on a circle. This means that the logarithmic growth will be absent where the translational invariance is broken. This happens for example for Dirichlet instead of periodic boundary condition.

\subsection{Disjoint intervals on each side}

In the following, we extend the above analysis to the case of two disjoint intervals on each side with a separation $d$. The numerical results about changing the inverse temperature $\beta$ and the mass $m$ are provided in appendix $\mathrm{A}$ and $\mathrm{B}$, respectively. In figure 5 , we investigate the time evolution of the normalized entanglement entropy $S_{\mathrm{EE}}$ (upper plots) and odd entanglement entropy $S_{\mathrm{OEE}}$ (lower plots) which are subtracted from the initial values for short-times (left plots) and long-times (right plots) in free massless scalar theory

\footnotetext{
${ }^{13}$ We would like to thank Erik Tonni for discussion about QFT limit.
} 

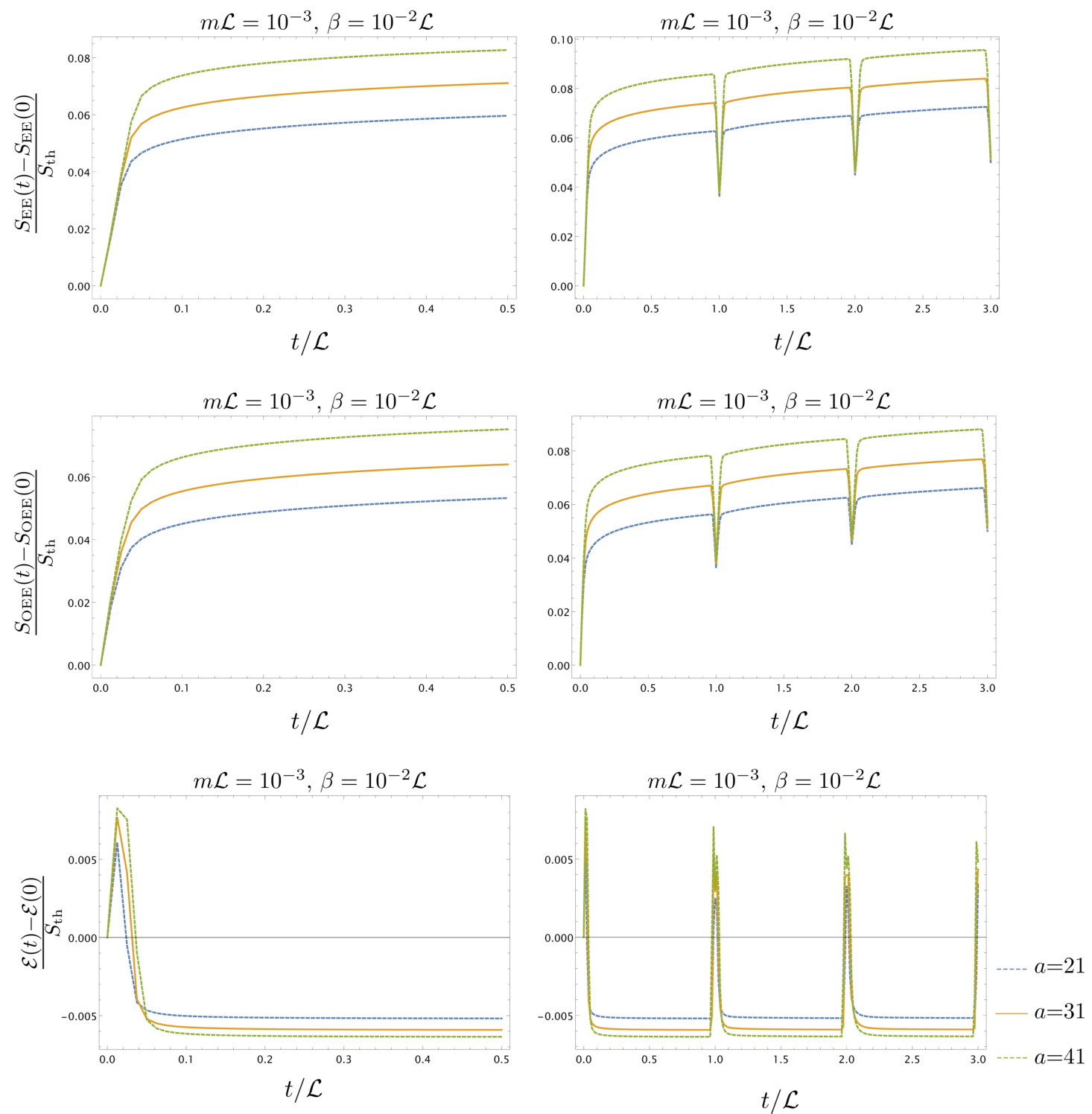

Figure 2. Time evolution of $S_{\mathrm{EE}}, S_{\mathrm{OEE}}$ and $\mathcal{E}$ in free massless scalar theory for various time regimes and various size of entangling regions, where the initial values are subtracted and the result is normalized with respect to the thermal entropy $S_{\mathrm{th}}$. We take $N=1501, N_{A_{L(R)}}=N_{A_{L_{1}\left(R_{1}\right)}}+N_{A_{L_{2}\left(R_{2}\right)}}=$ $20+a$. The upper-left plot denotes the growth of $S_{\mathrm{EE}}$ which is linear at times approximately equal to the size of an interval. The upper-right plot is for longer periods of time. One can see the finite size effects which induce a periodic behavior with periodicity $\mathcal{L}$. We see a logarithmic growth due to the presence of a zero-mode which we study further analytically in the next section. The middle-left and right panels denote the time dependency of $S_{\mathrm{OEE}}$. The effective time evolution of $S_{\mathrm{EE}}$ and $S_{\text {OEE }}$ are the same. The bottom-left plot denotes the short-time behavior of $\mathcal{E}$. In early times we see growth and then reduction with respect to the initial value of LN. The bottom-right plot is for a long time regime, in which the finite size effect exhibit itself as periodic behavior. 

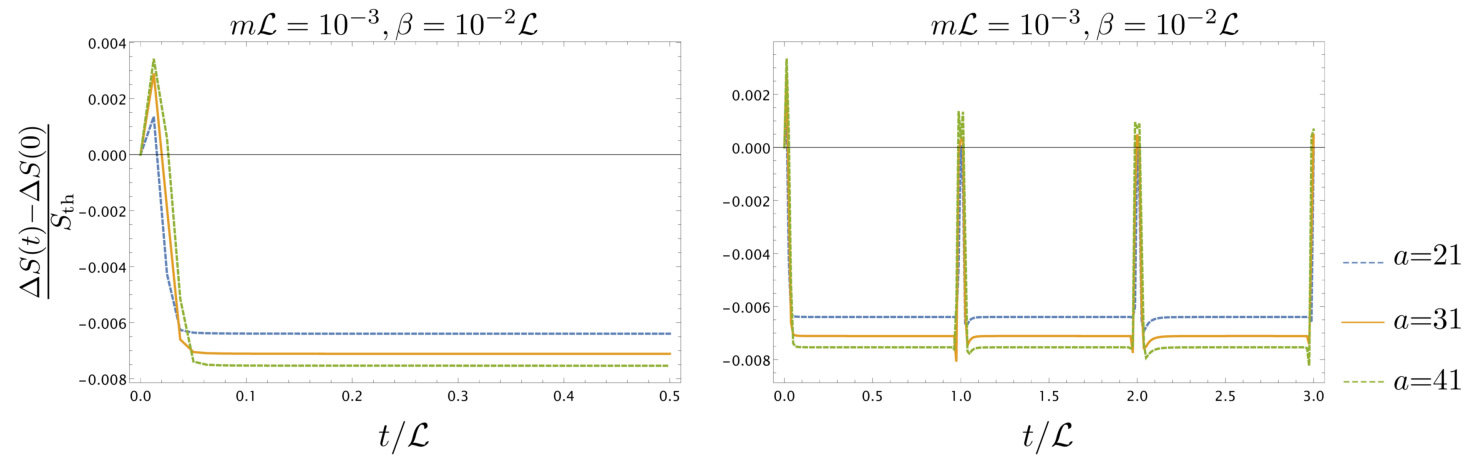

Figure 3. Time dependence of $\Delta S=S_{\mathrm{OEE}}-S_{\mathrm{EE}}$ in free massless scalar theory. We take $N=1501$, $N_{A_{L(R)}}=N_{A_{L_{1}\left(R_{1}\right)}}+N_{A_{L_{2}\left(R_{2}\right)}}=20+a$. Left panel corresponds to short-time scales and the right panel is for long-time scales.
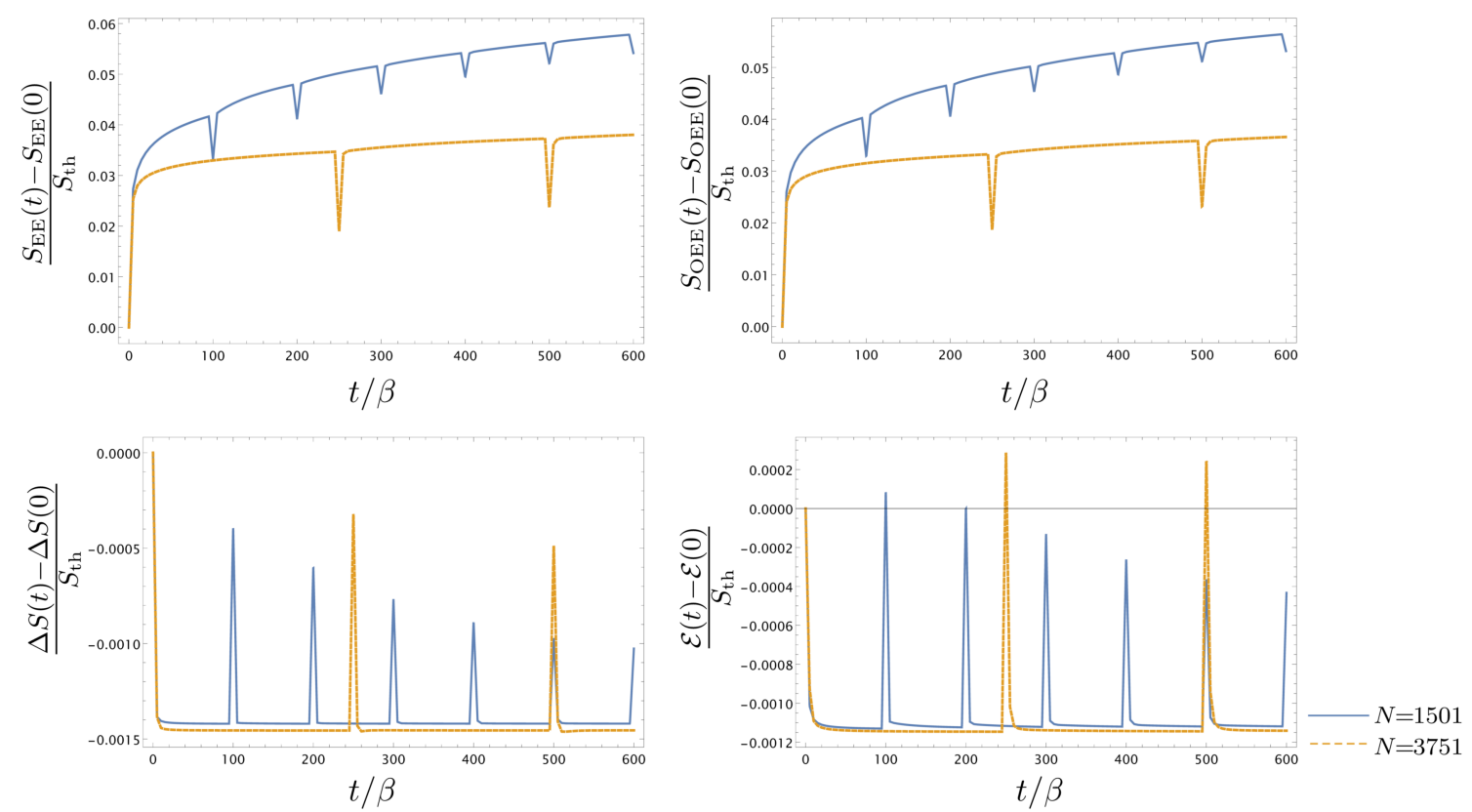

Figure 4. Decompactification limit: the dynamics of $S_{\mathrm{EE}}, S_{\mathrm{OEE}}, \Delta S=S_{\mathrm{OEE}}-S_{\mathrm{EE}}$ and $\mathcal{E}$ (subtracted from the initial values and normalized with thermal entropy) extrapolated from circle to a line with fixed lattice spacing $\delta$ and $m \beta=10^{-5}$. The blue curve denotes the theory on a circle with the total number of sites $N=1501$ and $N_{A_{L(R)}}=N_{A_{L_{1}\left(R_{1}\right)}}+N_{A_{L_{2}\left(R_{2}\right)}}=2+20$. The dashed-orange curve represents the same theory on a circle with fixing $\delta$ and increasing the total number of sites (and accordingly subsystem sites) to $N=3751$. Increasing $N$ causes the effects of finite size to be transferred to larger times. We also observe the logarithmic growth at the intermediate times. 

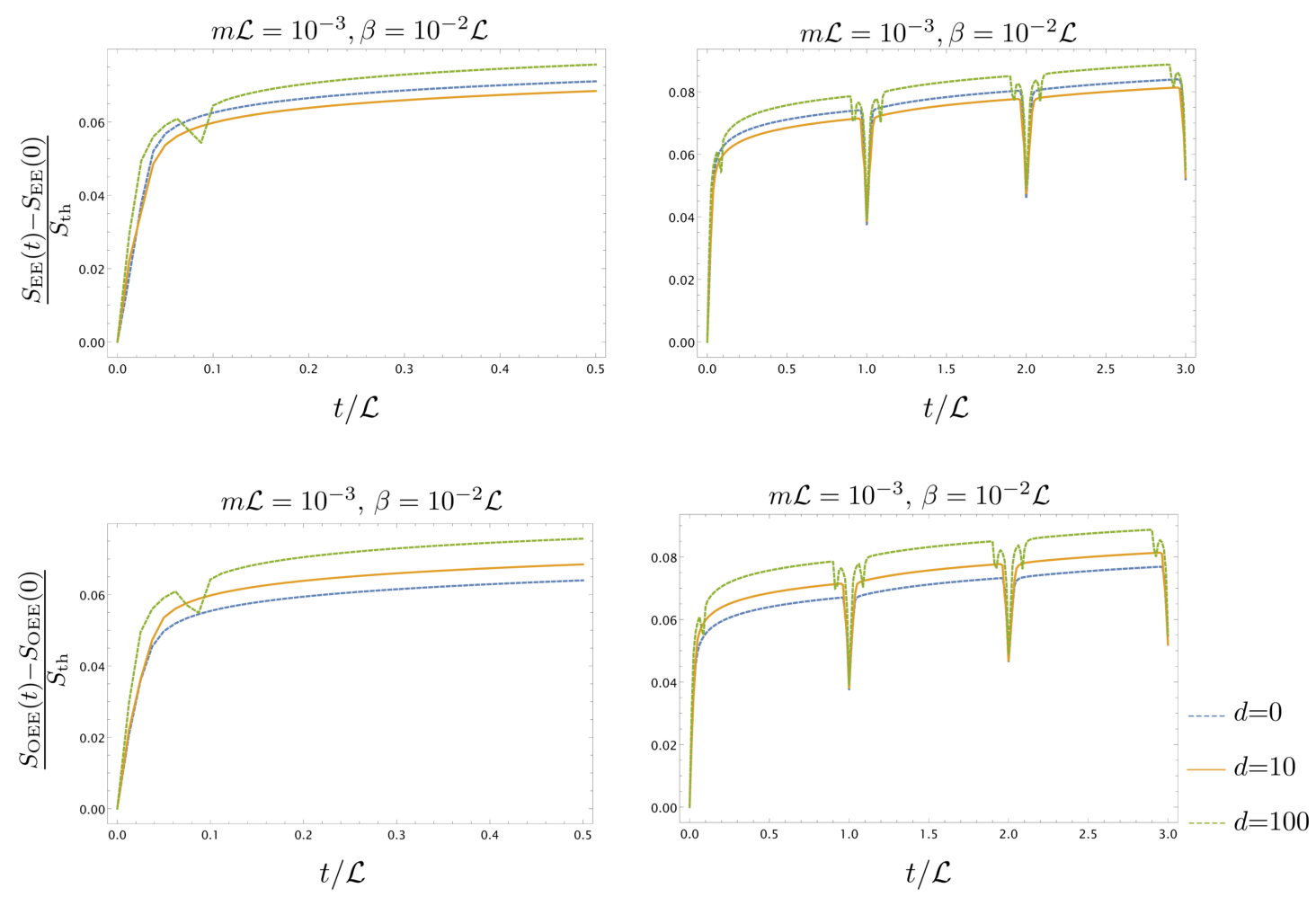

Figure 5. Disjoint intervals: time dependence of $S_{\mathrm{EE}}$ and $S_{\mathrm{OEE}}$ in free massless scalar theory, for various distances between entangling regions and various times. We take $N=1501, N_{A_{L(R)}}=$ $N_{A_{L_{1}\left(R_{1}\right)}}+N_{A_{L_{2}\left(R_{2}\right)}}=20+31$. Their effective evolution is the same in different times. One can see also the memory effect.

for different values of $d$. For short-times (times smaller than $\mathcal{L} / 2$ and in the absence of finite size effects), the growth of the $S_{\mathrm{EE}}$, as well as $S_{\mathrm{OEE}}$, is linear and lasts until approximately $t \sim l$; this is followed by saturation. For long-times (times larger than $\mathcal{L}-l$ where the finite size effects can be visible), the time dependence of $S_{\mathrm{EE}}$ (up-right panel), as well as $S_{\mathrm{OEE}}$ (bottom-right panel), is periodic with periodicity $\mathcal{L}$. Clearly, the pattern of evolution for $S_{\mathrm{EE}}$, as well as $S_{\mathrm{OEE}}$, is: linear growth for early times, $t \sim l$, a plateau of width approximately $\mathcal{L}-2 l$ in a middle timed and then a linear decrease up to order $t \sim l$. The oscillatory behavior can be observed for long times due to the finite size effect. Furthermore, we observe the memory effect [77] in $S_{\mathrm{EE}}$ and $S_{\mathrm{OEE}}$ for intervals $d>l$. The dip extends over the range $d / 2<t<d / 2+l$, and it is centered at $t=(d+l) / 2$. For the massive theory, the similar results are presented in appendix B, figure 17 . It is clear that for large masses, the oscillations are rather irregular due to the dephasing of the different kinds of quasi-particles with different group velocities. This dephasing also causes that the slope of linear growth at early times becomes significantly less than $2 S_{\mathrm{th}} / \mathcal{L}$. Another effect of changing mass can be seen in this figure where both $\mathrm{OEE}$ and EE fluctuate around the same value albeit we have increased the distance $d$. Of course this can be also understood by the quasi-particle picture where the entanglement increases, in general, by increasing the distance $d$ while it decreases by increasing the IR regulator mass $m$. 


\subsection{Different quasi-particles and different information quantities}

The figure 6 illustrates the time evolution of $\Delta S=S_{\mathrm{OEE}}-S_{\mathrm{EE}}$ (left panel) and the logarithmic negativity, $\mathcal{E}$ (right panel) which are normalized with respect to thermodynamic entropy $S_{\text {th }}$ and subtracted from their initial values. This figure together with figures $2,3,4$ and figures 12 and 13 in appendix A contain an interesting result: At least for free scalar $Q F T s$, the $\Delta S$ and $\mathcal{E}$ are qualitatively behave the same independent of the mass $m$, lattice spacing $\delta$, separation distance $d$ and inverse temperature $\beta .{ }^{14}$ These observations have two interesting consequences. Firstly, they imply that these two quantum information quantities $(\Delta S, \mathcal{E})$ are dual to the same geometric object in the bulk. Of course, there is a proposal for this $[22,23]$ but we were able to obtain new evidence to support it by studying the time-dependent TFD state.

Secondly, it is well-known that $[69,78]$ the logarithmic negativity is just a measure of entanglement (quantum correlation) between the subsystems $A_{1}$ and $A_{2}$ and the quasiparticles between $\left(A_{1}+A_{2}\right)$ and $B$ (figure 1$)$ do not contribute to it. To be more precise, all moments of partial transpose quasi-particles between $\left(A_{1}+A_{2}\right)$ and $B$ matter, but they cancel when taking the replica limit [79]. ${ }^{15}$ In contrast, the entanglement entropy is just affected by quasi-particles between $\left(A_{1}+A_{2}\right)$ and $B$. The time dependency of odd entanglement entropy is similar to the entanglement entropy but when we subtract it from entanglement entropy, interestingly, it behaves as logarithmic negativity. This implies that $S_{\mathrm{OEE}}$ not only has a contribution from the quasi-particles between $A_{1}$ and $A_{2}$ but also has contribution from quasi-particles between $\left(A_{1}+A_{2}\right)$ and $B$ in contrast to logarithmic negativity. ${ }^{16}$ Another interesting witness for this interpretation comes from figures 5 and 17. Accordingly, $S_{\mathrm{OEE}}$, in general, increases by increasing the distance $d$ but the situation is different for $S_{\mathrm{EE}}$. By increasing $d$, the $S_{\mathrm{EE}}$ firstly decreases but then increases. According to the above interpretation we can understand these different behaviors in the following way: for each pair of quasi-particles in the region outside the $\left(A_{1}+A_{2}\right)$, two events are possible: one quasi-particle travels to the region $A_{1}$ (or $A_{2}$ ) and another one remains in the outside region or one quasi-particle travels to the region $A_{1}$ and another one to the region $A_{2}$. The occurrence of the first event increases both the $S_{\mathrm{OEE}}$ and the $S_{\mathrm{EE}}$. But the occurrence of the second event increases the $S_{\mathrm{OEE}}$ but it decreases the $S_{\mathrm{EE}}$. Therefore, $S_{\mathrm{OEE}}$ is almost increasing but the behavior of $S_{\mathrm{EE}}$ crucially depends on the distance $d$ since the second event is more likely to occur for shorter distances $d$. One more witness comes from the observation of memory effect [77] in figures 5 and 17 when $d>l$. This effect can be understood by noting to the quasi-particles created at the midpoint between two intervals. Actually one quasi-particle entering the region $A_{1}$ and its partner entering the region $A_{2}$ around $t \sim d / 2$. When the dip exists, it extends over the range $d / 2<t<d / 2+l$ and it is centered at $t=(d+l) / 2$.

\footnotetext{
${ }^{14}$ For the disjoint intervals, we also checked the entanglement dynamics in the decompactification limit (or equivalently in the continuum limit): similar to the adjacent case, the effect of increasing $N$ appears in changing of oscillation period on the circle to larger times. For $S_{\mathrm{EE}}$ and $S_{\mathrm{OEE}}$, the coefficient of logarithmic growth also decreases by increasing the number of total sites $N$.

${ }^{15}$ We would like to thank Pasquale Calabrese for illuminating this point and also discussing our results.

${ }^{16}$ Of course this should be checked for a generic quantum state.
} 

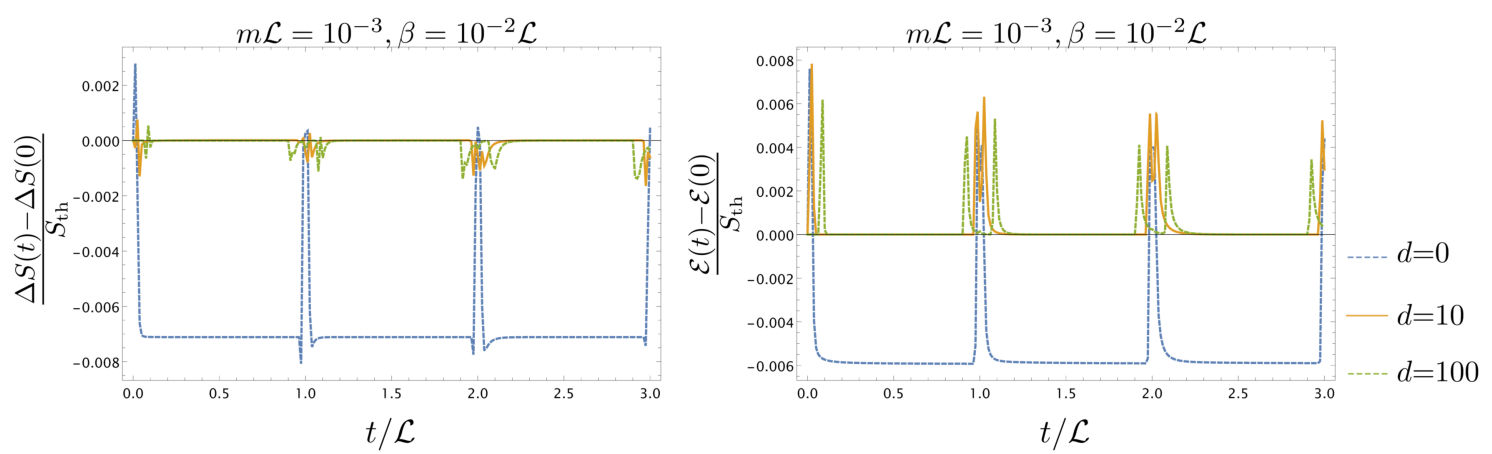

Figure 6. Long-time behavior of $\Delta S=S_{\mathrm{OEE}}-S_{\mathrm{EE}}$ and $\mathcal{E}$ in free massless scalar theory. We take $N=1501, N_{A_{L(R)}}=N_{A_{L_{1}\left(R_{1}\right)}}+N_{A_{L_{2}\left(R_{2}\right)}}=20+31$.
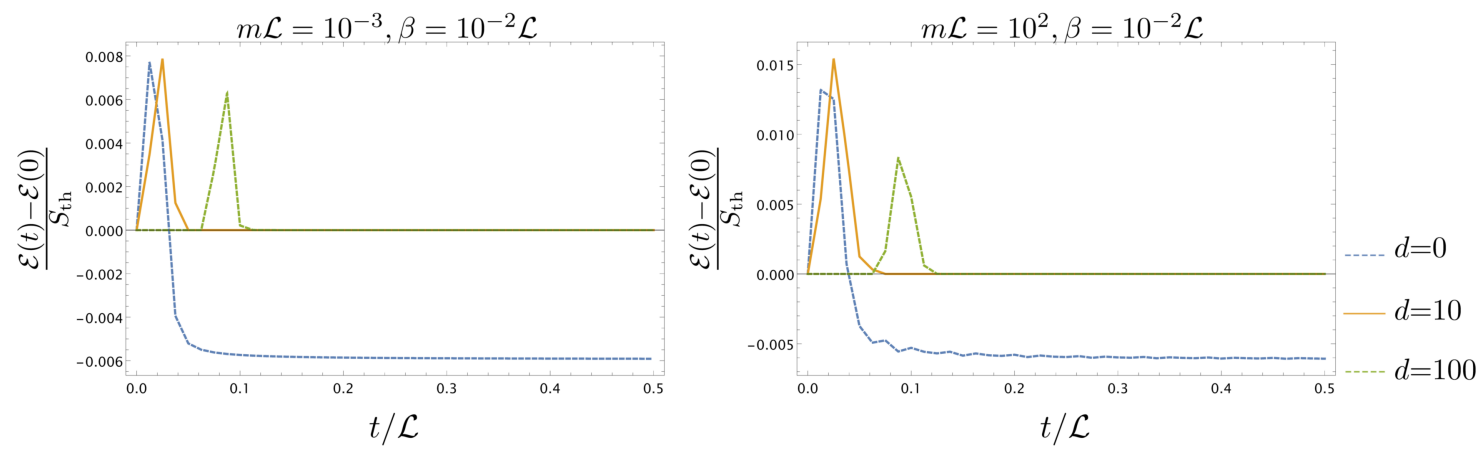

Figure 7. Early time behavior of $\mathcal{E}$ in free scalar theory. We take $N=1501, N_{A_{L(R)}}=N_{A_{L_{1}\left(R_{1}\right)}}+$ $N_{A_{L_{2}\left(R_{2}\right)}}=20+31$.

It is worth to mention that figure 6 contains an interesting information in very short times which is provided by figure 7 . It is clear that the LN is zero for times $t<d / 2$ and after that, it begins to grow linearly. This is in agreement with [44, 69]. A similar delay to start linear growth of $\Delta S=S_{\mathrm{OEE}}-S_{\mathrm{EE}}$ can also be seen in figure 6. Apart from that, the figure 7 contains more information. According to this figure, we observe that the logarithmic negativity is non-zero at early times if the size of subsystems is greater than the separation distance $d$. This is consistent with the behavior of the proposed dual geometric object i.e. with entanglement wedge cross-section. In the holographic prescription [22], whenever the separation between two intervals, $d$, is less than the size of subsystems, one has a non-zero entanglement wedge cross-section and by increasing $d$ a phase transition happens and entanglement wedge cross-section becomes zero.

\subsection{LN vs mutual and 1/2-Rényi mutual information}

For further investigation, we would like to study the time evolution of logarithmic negativity $\mathcal{E}$, mutual information $I, 1 / 2$-Rényi mutual information $I^{(1 / 2)}$ and explore their similarities and differences. The numerical results for two adjacent intervals are depicted in figure 8. The top-left panel denotes the time evolution of $\mathcal{E}$ and $I$ for long times and symmetric lengths for two subsystems. The top-right panel denotes the same quantities but with non-symmetric lengths for subsystems. In the symmetric case, at early times, 
$\mathcal{E}$ and $I$ have a very similar behavior: initial linear growth followed by an almost linear decreasing up to time $t \sim l_{1}\left(\equiv l_{A_{1}}\right)$. In this case, on time scales of the order of the system's size a difference between these quantities appears. In these time scales, the mutual information reaches a plateau while the logarithmic negativity decreases monotonically until the appearance of finite size effects. This observation implies that the decreasing behavior is a peculiarity of the entanglement and is not reflected by the correlation measures such as mutual information. This is in contrast to the holographic theories where the negativity is proportional to the mutual information for all times, i.e. $\mathcal{E}=3 I / 4$ [20] or $\mathcal{E}=I / 2$ [30]. Moreover, in the decompactification limit (increasing the system and subsystem sizes properly) the rate of decreasing of logarithmic negativity becomes more sharply which it might be the sign of sudden death of entanglement before the trivial (finite size effect) revival. ${ }^{17}$ Overall, the non-symmetric case has the same characteristics but with a difference which is the appearance of a (narrow) plateau after the first linear growth, for both the logarithmic negativity and mutual information. All these are in agreement with the results of [69] which is in a different setup. The bottom plot denotes the time evolution of $\mathcal{E}$ (solid curves) and $1 / 2$-Rényi mutual information $I^{(1 / 2)}$ (dotted-dashed curves). The initial growth which is linear and then decreasing followed by saturation is approximately the same between them which is in agreement with [44]. This similar behavior implies an interesting unification of these two seemingly different information-theoretic quantities in integrable models. i.e. $\mathcal{E} \sim I^{(1 / 2)}$. Since this relation crucially related to the existence of infinitely-living quasiparticle excitations, it might breakdown in chaotic (non-integrable) models.

\subsection{Some inequalities for OEE}

Before closing this section, it is worth studying several proposed inequality for odd entanglement entropy $S_{\mathrm{OEE}}[31]$. We can check numerically some of these inequalities: ${ }^{18}$

- $S_{\mathrm{OEE}}(A: B) \geq 0$ (positive semi-definiteness)

- $S_{\mathrm{OEE}}\left(A: B_{1} B_{2}\right) \geq S_{\mathrm{OEE}}\left(A: B_{1}\right)$ (monotonicity)

- $S_{\mathrm{OEE}}\left(A_{1}: A_{2}\right) \geq \max \left[S_{\mathrm{EE}}\left(A_{1}\right), S_{\mathrm{EE}}\left(A_{2}\right)\right]$

According to the results of this section, the positive semi-definiteness clearly is established. Monotonicity relation means that by enlarging one of two subsystems, the total amount of correlations between the two subsystems increases. According to figure 2, it is clear that both of $S_{\mathrm{EE}}$ and $S_{\mathrm{OEE}}$ satisfies the monotonicity relation. In figure 9 , we can also simply see that the $S_{\mathrm{OEE}}$ satisfies the last inequality. In this figure, $S_{\mathrm{EE}}(t)$ refers to the greater one between $S_{\mathrm{EE}}\left(A_{1}\right)$ and $S_{\mathrm{EE}}\left(A_{2}\right)$.

\section{$5 \quad$ Logarithmic growth at intermediate times}

In the previous section, we have observed the existence of a logarithmic growth regime for odd entanglement entropy, instead of a sharp saturation, at intermediate times. In the case

\footnotetext{
${ }^{17}$ The same phenomenon is explored in different quench setup [69] and for other entanglement measures [80]. Of course it might be the lattice effect and will absent in true continuous QFT [81].

${ }^{18}$ We would like to thank Kotaro Tamaoka for pointing out the last inequality to us.
} 

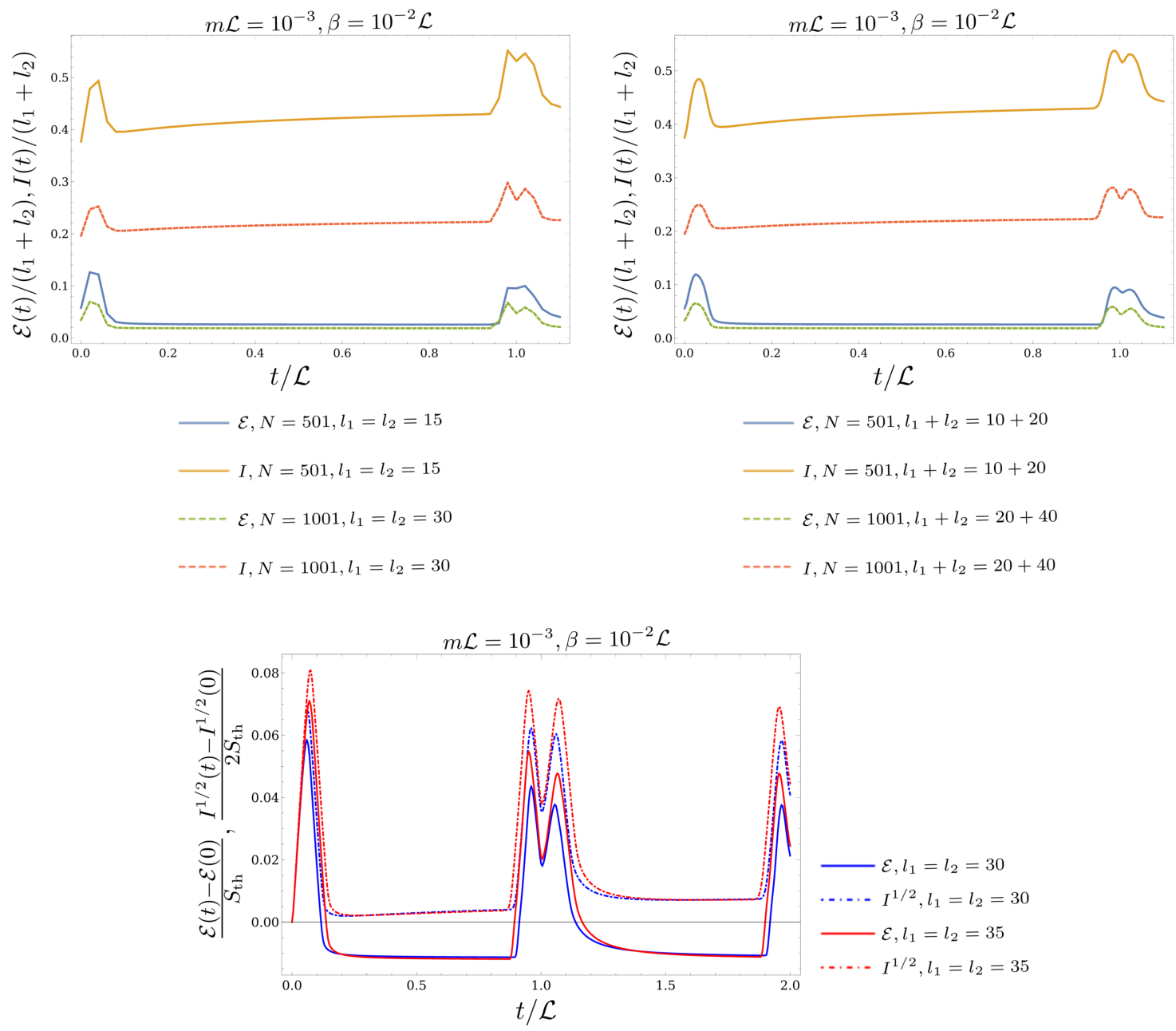

Figure 8. Time dependency of $\mathcal{E}$ and mutual information $I$ are presented in top panel. The topleft and top-right one are for symmetric and non-symmetric subsystems, respectively. The bottom panel demonstrates the time evolution of $\mathcal{E}$ and 1/2-Rényi mutual information $I^{(1 / 2)}$ for $N=501$.
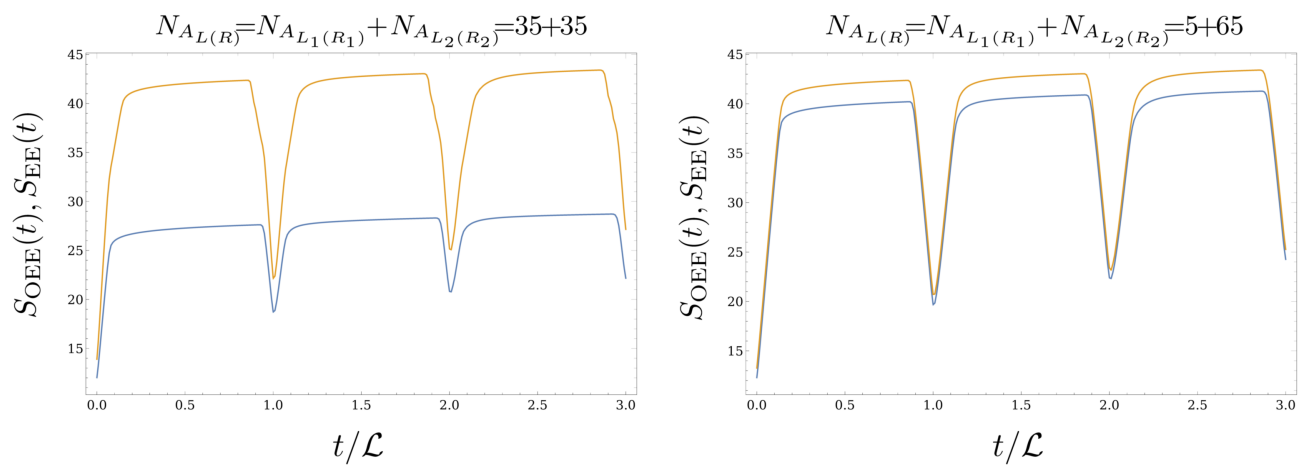

Figure 9. The $S_{\mathrm{EE}}$ (blue) and $S_{\mathrm{OEE}}\left(\right.$ orange) are presented for $N=501, m \mathcal{L}=10^{-3}, d=0, \beta=10^{-2} \mathcal{L}$. 
of entanglement entropy of a pure state, this behavior was observed previously $[9,13,58]$. This behavior, as we will discuss more in the following, is due to the presence of a zero-mode, namely the momentum mode with $k=0$ in the massless limit. It is worth emphasizing that this gapless zero-mode does not lead to a ballistic propagation as in the quasi-particle picture, but it instead has a diffusive nature [58]. ${ }^{19}$ The authors of [13] have studied the logarithmic contribution based on analyzing the entanglement entropy for a single degree of freedom on each side of TFD in the massless limit, i.e., $m \rightarrow 0$, and found that

$$
S_{\mathrm{EE}}(t) \sim \begin{cases}\frac{1}{2} \log (t / \mathcal{L}) & \delta \ll t<\mathcal{L}, \\ \log (t / \mathcal{L}) & \mathcal{L}<t \ll m^{-1}, \\ \log |\sin (m t)| & \mathcal{L}<t .\end{cases}
$$

In order to proceed for odd entanglement entropy, we will follow [13] and determine the consequences of the existence of the zero-mode analytically. We will take a single site in each entangling region, and derive the full asymptotic behavior of odd entanglement entropy in the limit $\beta, m^{-1} \ll \mathcal{L}$, which will turn out to be the same as (5.1). When we extend the analysis for logarithmic negativity, no logarithmic correction will be observed. It is worth noting that in order to make the study of this logarithmic behavior feasible, we will focus on a subsystem consisting of a single lattice site on each side of the TFD state. Accordingly, we will intentionally suppress the linear regime by choosing a subsystem size that vanishes in the continuum limit. Then, we will extend the observed asymptotic behavior to the case of larger subsystems due to the fact that the zero-mode is completely non-local and therefore affects local subsystems in a similar way, regardless of their size. We should also emphasize that this expression will fail to describe the entanglement accurately in the regime $t<\delta$ in which the precise form of oscillations of various modes will determine the time evolution.

For highly entangled states, the von Neumann entropy $S_{\mathrm{EE}}\left(\rho_{A_{12}}(t)\right)$ can be approximated as $[57,59]^{20}$

$$
S_{\mathrm{EE}}\left(\rho_{A_{12}}(t)\right) \sim S_{2}\left(\rho_{A_{12}}(t)\right)+2 N_{A_{12}}(1-2 \log 2),
$$

where $S_{2}\left(\rho_{A_{12}}(t)\right)$ is the second Rényi entropy,

$$
S_{2}\left(\rho_{A_{12}}(t)\right)=\frac{1}{2} \log \left(\operatorname{det}\left(G_{A_{12}}(t)\right)\right) .
$$

Using (3.13), we can find a similar upper bound formula for the odd entanglement entropy. Actually, instead of $G_{A_{12}}(t)$ we use $\tilde{G}_{A_{12}}(t)$ and approximate the $S_{\mathrm{OEE}}$ for a single-mode on each side with the modified second Rényi entropy, $\tilde{S}_{2}$. As we discussed in section 3 , in order to find $\tilde{G}_{12}^{a b}$, one first need to find the covariance matrix, $G_{12}^{a b}$, and then take a partial transpose with respect to the momentum degrees of freedom in the $A_{2}$ subregion. This can

\footnotetext{
${ }^{19}$ The authors have considered both a boundary state quench and a global mass quench. For other relevant zero-mode effect discussions see refs [82, 83].

${ }^{20}$ The error scales as $\exp \left(-2 S_{2}\right)$. Therefore, it decreases exponentially for highly entangled states [13].
} 
be accomplished by acting with the time-reversal operator $\mathcal{R}_{A_{2}}$,

$$
\tilde{G}_{12}^{a b}=\mathcal{R}_{A_{2}} \cdot G_{12}^{a b} \cdot \mathcal{R}_{A_{2}},
$$

where $\mathcal{R}_{A_{2}}$ is a square matrix of length $2 N_{A} \times 2 N_{A}$,

$$
\mathcal{R}_{A_{2}}=\operatorname{diag}\{1,1, \cdots, 1,-1, \cdots,-1\}
$$

and the number of -1 elements is equal to the length of subregion $A_{2}$. Here, $N_{A_{1}} \equiv$ $N_{A_{R_{1}}}=1$ and $N_{A_{2}} \equiv N_{A_{L_{1}}}=1$, so we have

$$
\operatorname{det}\left(\tilde{G}_{12}^{a b}\right)=\operatorname{det}\left(\mathcal{R}_{A_{2}} \cdot G_{12}^{a b} \cdot \mathcal{R}_{A_{2}}\right)=\operatorname{det}\left(G_{12}^{a b}\right) .
$$

Note that with respect to this specific choice of subsystems, one obtains $\operatorname{det}\left(\tilde{G}_{12}^{a b}\right)=$ $\operatorname{det}\left(G_{12}^{a b}\right)$. Therefore, we can use the second Rényi entropy itself for evaluating the zeromode effect on the evolution of $S_{\mathrm{OEE}}$. In the following, we explicitly compute it.

The covariance matrix, $G_{A}(t)$, associated to subregion with a single site at position $x$ on both sides of the TFD, with respect to the dimensionless basis $\tilde{\xi}_{k}^{a}=\left(\tilde{q}_{k}^{L}, \tilde{q}_{k}^{R}, \tilde{p}_{k}^{L}, \tilde{p}_{k}^{R}\right){ }^{21}$ is given as follows:

$$
\begin{aligned}
\tilde{G}_{k}^{a b}(t) & =\left\langle\operatorname{TFD}(t)\left|\tilde{\xi}_{k}^{a} \tilde{\xi}_{k}^{\dagger b}+\tilde{\xi}_{k}^{\dagger b} \tilde{\xi}_{k}^{a}\right| \operatorname{TFD}(t)\right\rangle \\
& =\left(\begin{array}{cccc}
\frac{\cosh \left(2 \alpha_{k}\right)}{\lambda_{k}} & \frac{\cos \left(\omega_{k} t\right) \sinh \left(2 \alpha_{k}\right)}{\lambda_{k}} & 0 & -\sin \left(\omega_{k} t\right) \sinh \left(2 \alpha_{k}\right) \\
\frac{\cos \left(\omega_{k} t\right) \sinh \left(2 \alpha_{k}\right)}{\lambda_{k}} & \frac{\cosh \left(2 \alpha_{k}\right)}{\lambda_{k}} & -\sin \left(\omega_{k} t\right) \sinh \left(2 \alpha_{k}\right) & 0 \\
0 & -\sin \left(\omega_{k} t\right) \sinh \left(2 \alpha_{k}\right) & \lambda_{k} \cosh \left(2 \alpha_{k}\right) & -\lambda_{k} \cos \left(\omega_{k} t\right) \sinh \left(2 \alpha_{k}\right) \\
-\sin \left(\omega_{k} t\right) \sinh \left(2 \alpha_{k}\right) & 0 & -\lambda_{k} \cos \left(\omega_{k} t\right) \sinh \left(2 \alpha_{k}\right) & \lambda_{k} \cosh \left(2 \alpha_{k}\right)
\end{array}\right),
\end{aligned}
$$

where,

$$
\omega_{k}=\sqrt{m^{2}+\frac{4}{\delta^{2}} \sin ^{2}\left(\frac{\pi k}{N}\right)}, \quad \alpha_{k}=\frac{1}{2} \log \operatorname{coth}\left(\frac{\beta \omega_{k}}{4}\right), \quad \lambda_{k}=\omega_{k} \mathcal{L} .
$$

By using the inverse Fourier transformation $\xi_{x}^{a}=\frac{1}{\sqrt{N}} \sum_{k=1}^{N} e^{-\frac{2 \pi i k x}{N}} \tilde{\xi}_{k}^{a}$, in the position basis $\xi_{x}^{a}=\left(q_{x}^{L}, q_{x}^{R}, p_{x}^{L}, p_{x}^{R}\right)$, we have

$$
\begin{aligned}
G_{x, y}^{a b}= & \frac{1}{N} \sum_{k} e^{-\frac{2 \pi i k(x-y)}{N}} G_{k}^{a b} \\
= & \frac{1}{N} \sum_{k} e^{-\frac{2 \pi i k(x-y)}{N}} \\
& \times\left(\begin{array}{cccc}
\frac{\cosh \left(2 \alpha_{k}\right)}{\lambda_{k}} & \frac{\cos \left(\omega_{\mu k} t\right) \sinh \left(2 \alpha_{k}\right)}{\lambda_{k}} & 0 & -\sin \left(\omega_{k} t\right) \sinh \left(2 \alpha_{k}\right) \\
\frac{\cos \left(\omega_{k} t\right) \sinh \left(2 \alpha_{k}\right)}{\lambda_{k}} & \frac{\cosh \left(2 \alpha_{k}\right)}{\lambda_{k}} & -\sin \left(\omega_{k} t\right) \sinh \left(2 \alpha_{k}\right) & 0 \\
0 & -\sin \left(\omega_{k} t\right) \sinh \left(2 \alpha_{k}\right) & \lambda_{k} \cosh \left(2 \alpha_{k}\right) & -\lambda_{k} \cos \left(\omega_{k} t\right) \sinh \left(2 \alpha_{k}\right) \\
-\sin \left(\omega_{k} t\right) \sinh \left(2 \alpha_{k}\right) & 0 & -\lambda_{k} \cos \left(\omega_{k} t\right) \sinh \left(2 \alpha_{k}\right) & \lambda_{k} \cosh \left(2 \alpha_{k}\right)
\end{array}\right) .
\end{aligned}
$$

\footnotetext{
${ }^{21}$ These variables are related to variables of momentum space dual to the discretized field according to $q_{a}=\frac{1}{\sqrt{N}} \Phi\left(x_{a}\right)$ and $p_{a}=\frac{\mathcal{L}}{\sqrt{N}} \Pi\left(x_{a}\right)$.
} 
By setting $x=y$, we can obtain the odd entanglement entropy of a subsystem consisting of a single degree of freedom on each left and right side of the TFD. By studying the asymptotic behavior of odd entanglement entropy, we can identify the contribution of the zero-mode in the massless limit, $m \rightarrow 0$; this exhibits itself as the logarithmic term. According to the above explanations, we can act as follows: setting $x=y$ in the (5.9) gives:

$$
\begin{aligned}
G_{x, x}^{a b}(t)= & \frac{1}{N} \sum_{k=0}^{N-1} \\
& \times\left(\begin{array}{cccc}
\frac{\cosh \left(2 \alpha_{k}\right)}{\lambda_{k}} & \frac{\cos \left(\omega_{k} t\right) \sinh \left(2 \alpha_{k}\right)}{\lambda_{k}} & 0 & -\sin \left(\omega_{k} t\right) \sinh \left(2 \alpha_{k}\right) \\
\frac{\cos \left(\omega_{k} t\right) \sinh \left(2 \alpha_{k}\right)}{\lambda_{k}} & \frac{\cosh \left(2 \alpha_{k}\right)}{\lambda_{k}} & -\sin \left(\omega_{k} t\right) \sinh \left(2 \alpha_{k}\right) & 0 \\
0 & -\sin \left(\omega_{k} t\right) \sinh \left(2 \alpha_{k}\right) & \lambda_{k} \cosh \left(2 \alpha_{k}\right) & -\lambda_{k} \cos \left(\omega_{k} t\right) \sinh \left(2 \alpha_{k}\right) \\
-\sin \left(\omega_{k} t\right) \sinh \left(2 \alpha_{k}\right) & 0 & -\lambda_{k} \cos \left(\omega_{k} t\right) \sinh \left(2 \alpha_{k}\right) & \lambda_{k} \cosh \left(2 \alpha_{k}\right)
\end{array}\right) .
\end{aligned}
$$

Considering (5.2), in order to compute the odd entanglement entropy, one needs to evaluate the second Rényi entropy which from (5.3) is related to the determinant in (5.10), recall $\operatorname{det}\left(\tilde{G}_{12}^{a b}\right)=\operatorname{det}\left(G_{12}^{a b}\right)$. The details of this calculation is postponed to appendix $\mathrm{C}$ and we only report the final result here. In the range $t \gg \delta=\mathcal{L} / N$ and in the limit $N \rightarrow \infty$, $m \mathcal{L} \ll 1, \beta / \mathcal{L} \ll 1$ and $m \ll \delta^{-1}$, the odd entanglement entropy becomes:

$$
S_{\mathrm{OEE}}\left(\rho_{A}(t)\right) \sim 2(1-2 \log 2)+\log \left(\frac{2 e_{2}}{N \beta m}\right)+\frac{1}{2} \log \left[\frac{\sin ^{2}(m t)+Q}{m^{2} \mathcal{L}^{2}}\right],
$$

where $Q$ is defined by

$$
Q=\frac{m^{2} \mathcal{L}^{2}}{\pi^{2}} \sum_{k=1}^{\infty} \frac{1-\cos \left[\left(\frac{2 \pi k}{\mathcal{L}}\right) t\right]}{k^{2}}
$$

and $e_{2}$ is given in equation (C.6). The logarithmic term in the (5.11) can be simplified in the three regimes as follows,

$$
\frac{1}{2} \log \left[\frac{\sin ^{2}(m t)+Q}{m^{2} \mathcal{L}^{2}}\right] \sim \begin{cases}\frac{1}{2} \log (t / \mathcal{L}) & \delta \ll t<\mathcal{L} \\ \log (t / \mathcal{L}) & \mathcal{L}<t \ll m^{-1} \\ \log \left(\frac{|\sin (m t)|}{m \mathcal{L}}\right) & \mathcal{L} \ll t\end{cases}
$$

Hence, the asymptotic form of the odd entanglement entropy in these regimes is given by

$$
S_{\mathrm{OEE}}\left(\rho_{A}(t)\right) \sim 2(1-2 \log 2)+\log \left(\frac{2 e_{2}}{N \beta m}\right)+ \begin{cases}\frac{1}{2} \log (t / \mathcal{L}) & \delta \ll t<\mathcal{L}, \\ \log (t / \mathcal{L}) & \mathcal{L}<t \ll m^{-1}, \\ \log \left(\frac{|\sin (m t)|}{m \mathcal{L}}\right) & \mathcal{L} \ll t,\end{cases}
$$

which matches with our numerical results (see below). Of course, the second case is simply derived by the third case under the condition $t \ll m^{-1}$. In the large time asymptotics, the 
behavior is oscillatory with frequency $m$. This is expected due to the upper bound for the growth of the entanglement which is provided by the thermal state. Hence, only for times $t \ll m^{-1}$ we have the logarithmic behavior and for longer times we have the oscillatory behavior with frequency $m$. It is worth noting that in order to determine the entanglement evolution in the regime $t<\delta$ we must account for the precise form of oscillations of various modes, hence, the above expression will fail in that regime.

In the following, we present the numerically evaluated $S_{\mathrm{EE}}, S_{\mathrm{OEE}}$ and $\mathcal{E}$ for a single site on each side of TFD in the limit $m \rightarrow 0$ and find a logarithmic contribution for $S_{\mathrm{OEE}}$ which is due to the presence of a zero-mode and compare it with analytical result, (5.14). In figure 10, the analytical and numerical results are illustrated for the $S_{\mathrm{EE}}$ and $S_{\mathrm{OEE}}$ in the top panels. The $\mathcal{E}$ is plotted in the bottom panel. The logarithmic growth for $S_{\mathrm{OEE}}$ is observed in variant time scales with different coefficients and it well-matches with the analytical result (5.14). It is worth to mention that we also observe similar behavior for the case where $N_{A_{L(R)}}=N_{A_{L_{1}\left(R_{1}\right)}}+N_{A_{L_{2}\left(R_{2}\right)}}=1+1$, in the massless limit. Since the entanglement of two sites does not have a well-defined continuum limit therefore in this figure we do not divide the result by the thermodynamic entropy $S_{\mathrm{th}}$.

Moreover, according to the figure 10, the entanglement entropy and odd entanglement entropy became equal while the logarithmic negativity vanishes. This implies that the logarithmic negativity is proportional to $\Delta S=S_{\mathrm{OEE}}-S_{\mathrm{EE}}$ and this is in agreement with the result of [24]. At the analytical level, this comes from the fact that the modified reduced density matrix $\tilde{G}_{A_{12}}(t)$ and reduced density matrix $G_{A_{12}}(t)$ are equal, see equation (5.6). More evidence has been provided for the proportionality of $\Delta S$ and logarithmic negativity in subsection 4.1 (figure 3 and bottom plot in figure 2).

\section{Conclusion}

In this manuscript, we have studied entanglement dynamics of TFD state for $1+1$ dimensional free scalar theory on a lattice by considering entanglement entropy, odd entanglement entropy and logarithmic negativity. The scalar field is discretized on two circles each with length $\mathcal{L}$ and the entangled subsystems are non-complementary regions each with two adjacent or two disjoint subregions. To compute desired entanglement measures we have used the covariance matrix formalism which is proper for Gaussian states. For evaluating entanglement entropy the eigenvalues of reduced covariance matrix are needed however for odd entanglement entropy and logarithmic negativity the eigenvalues of partial transposed reduced covariance matrix are required. It is worth to mention that in the spirit of [52] our setup is named an unusual quantum quench scenario [13] in which two decoupled subsystems are entangled via their initial conditions.

The general perspective for time evaluation of entanglement entropy and odd entanglement entropy is an early linear growth then saturation for short times and then oscillatory behavior for longer times with periodicity of order the circle size $\mathcal{L}$. Also, for massless theory we have observed a logarithmic growth for the intermediate times due to presence of a zero-mode. However, this growth is limited by the upper bounds 3.17 and 3.18. The linear growth, saturation and oscillatory behavior can be understood using the quasi-particle pic- 

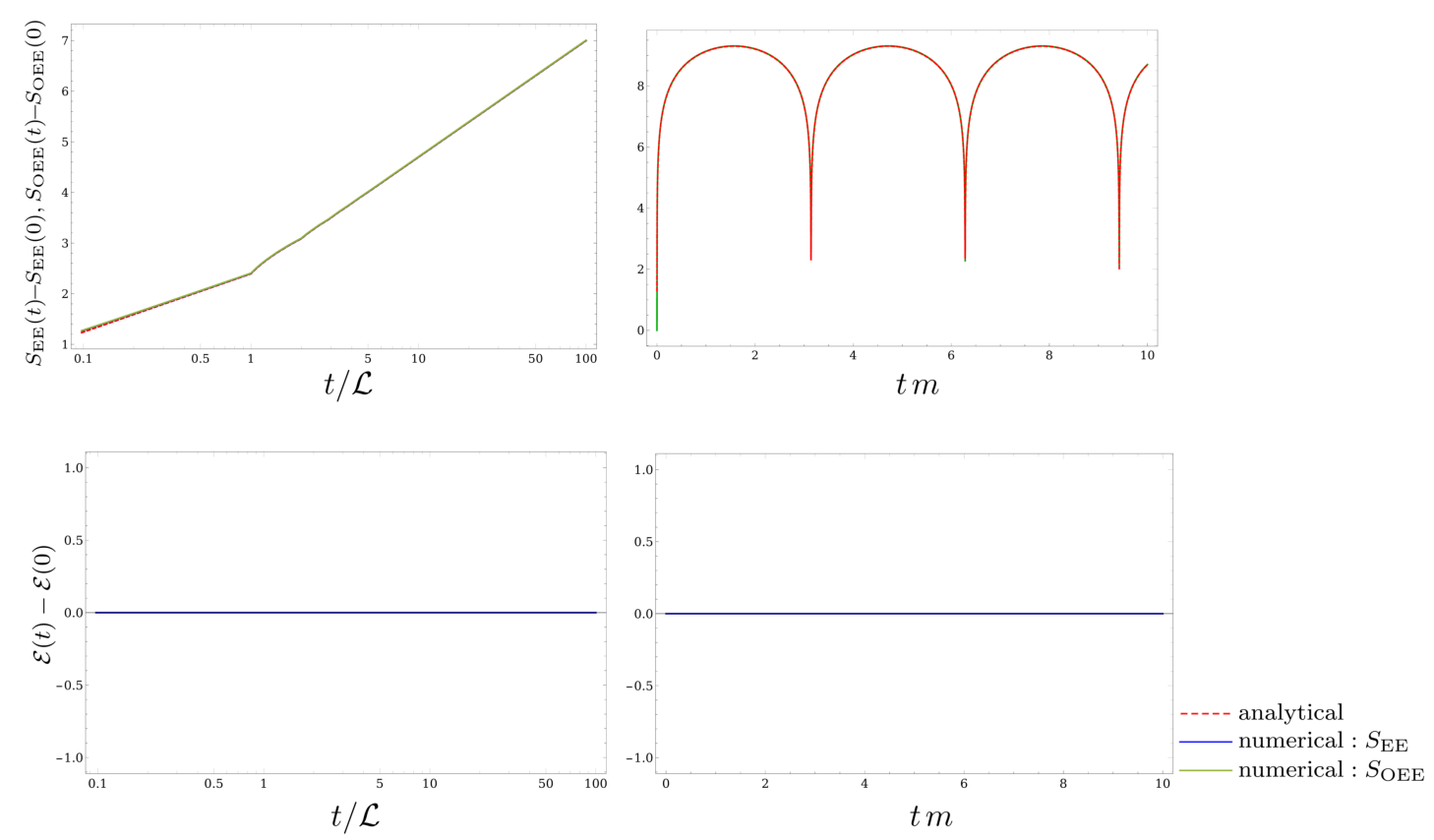

Figure 10. These plots correspond to the case of a subsystem consisting of a single site on each side for $m=10^{-3} / \mathcal{L}, \beta=10^{-2} \mathcal{L}$ and the total sites $N=1501$. We compare the analytical and numerical results for $S_{\mathrm{EE}}, S_{\mathrm{OEE}}$ and $\mathcal{E}$. In the top-right panel, the periodic behavior with respect to the parameter $t m$ is clear.

ture of $[7,8]$. According to the quasi-particle picture, the pre-quench initial state $\left|\psi_{0}\right\rangle$ acts as a source for independent entangled pairs each with an effective group velocity $v_{n}(1.2)$ that move ballistically in opposite directions through the system. The entanglement can spread when one quasi-particle is inside the subsystem and its partner is outside. The initially linear growth arises due to the flux of quasi-particles going out of the interval while their partners are still inside. This linear growth lasts until times $t \sim l(l$ is the size of the entangling region) then it saturates at a value that is proportional to the thermal entropy of the system. Moreover, the time dependence of quantum correlations exhibits an oscillatory behavior periodically equal to the circle circumference, $\mathcal{L}$, due to finite size effects. This can also be understood by a quasi-particle picture where meeting quasi-particles on the opposite side of the circle leads to reducing the correlations between the subsystem and its complement. We observed a regular oscillation in the massless limit since all the quasi-particles effectively move with the speed of light while for larger masses the different quasi-particles have different group velocities which lead to irregular oscillation. ${ }^{22}$ Besides, we have found that correlations can be increased by increasing both the separation $d$ and the temperature. According to the quasi-particle picture, for the greater separation $d$, more quasi-particle pairs are produced in the region between the two subsystems which leads to increasing the share of pairs that contribute to entanglement. Also enhancement by increasing the temperature indicates that odd entanglement entropy same as entangle-

\footnotetext{
${ }^{22}$ Actually, the quasi-particle picture provide a universal description for integrable models and it fails to capture dynamics of entanglement in more generic systems such as chaotic ones.
} 
ment entropy is a measure of both classical and quantum correlations. To investigate the entanglement dynamics in the continuum limit, the lattice spacing $\delta$, mass $m$ and inverse temperature $\beta$ are fixed but the total number of lattice sites $N$ is increased. Effectively, in this limit the period of oscillation becomes larger and zero-mode contribution becomes less important at intermediate times. Furthermore, there are several unproved inequalities for odd entanglement entropy [31] where we have confirmed some of them numerically.

It is worth emphasizing again that the logarithmic growth of odd entanglement entropy (and also entanglement entropy) can not be understood using the quasi-particle picture. To find a qualitative description and for tractability of computations, we focused on a subsystem consisting of a single site on each side of the TFD state. Accordingly, we intentionally suppress the linear regime by choosing a subsystem size that vanishes in the continuum limit. But we can restore the original system since zero-mode is non-local and can affect local subsystems, regardless of their size. In another word, zero-mode contribution is additive. The analytical results is derived by the approach of [13], which is based on a relation between the entanglement entropy and Rényi entropy of order 2 that is held for Gaussian states. By this approach, the Rényi entropy of order 2 for a configuration with a single site on each side can be evaluated by the determinant of a 4 -by- 4 covariance matrix and therefore its time dependency simply can be analyzed analytically. Interestingly, in our special decomposition of the subsystem, the determinants of the modified reduced density matrix $\tilde{G}_{A_{12}}(t)$ and reduced density matrix $G_{A_{12}}(t)$ became equal therefore evaluation of odd entanglement entropy become same as entanglement entropy. ${ }^{23}$ The time evolution pattern is composed of three time regimes: the first logarithmic regime: $S_{\mathrm{OEE}}\left(\rho_{A}(t)\right) \sim \frac{1}{2} \log (t / \mathcal{L})$ for $t<\mathcal{L}$, a second logarithmic region: $S_{\mathrm{OEE}}\left(\rho_{A}(t)\right) \sim \log (t / \mathcal{L})$ for $\mathcal{L}<t \ll m^{-1}$ and finally an oscillating regime: $S_{\mathrm{OEE}}\left(\rho_{A}(t)\right) \sim \log (\sin m t)$ when $t$ is of the same order as $m^{-1}$.

Apart from the mentioned results, we have also studied logarithmic negativity for symmetric and non-symmetric subsystems $A_{1}, A_{2}$. In the symmetric case, at early times, it exists initial linear growth followed by an almost linear decreasing up to revival time. This is in agreement with previous studies $[44,69]$ in scaling limit ${ }^{24}$ and different setup. By comparison with mutual information in the same setup, we have discussed that this implies that the decreasing behavior is a peculiarity of the entanglement and is not reflected by the correlation measures. Moreover, in the decompactification limit the rate of decreasing of logarithmic negativity becomes more sharply which it might be the sign of sudden death of entanglement before the trivial (finite size effect) revival. Overall, the non-symmetric case has the same characteristics but with a difference which is the appearance of a (narrow) plateau after an early linear growth. We have also observed that whenever the size of subsystems is greater than separation distance $d$, logarithmic negativity is non-zero for early times. It is consistent with a dual holographic picture, in which logarithmic negativity is dual to the geometric object named as entanglement wedge cross-section. In the holographic prescription, for two disjoint intervals, whenever the disjoint separation between two intervals $d$ is less than the size of subsystems, one has a non-zero entanglement wedge cross-section and when $d$ increases one can see a phase transition via vanishing the

\footnotetext{
${ }^{23}$ For entanglement entropy, see [13].

${ }^{24}$ The scaling limit means the long times and large subsystems with their ratio fixed.
} 
entanglement wedge cross-section. On the other hand, according to the result of [24], the logarithmic negativity is proportional to $\Delta S=S_{\mathrm{OEE}}-S_{\mathrm{EE}}$. Therefore, whenever logarithmic negativity vanishes, the entanglement entropy and odd entanglement entropy became equal which also matches with our numerical results. Moreover, for the adjacent case, we observe that by decreasing the temperature the delay time for starting the growth of logarithmic negativity becomes larger.

For future direction, it would be very interesting to obtain analytical form for evaluation of odd entanglement entropy since our results provide evidence that it should exist. We observed that the difference between odd entanglement entropy and entanglement entropy is proportional to the logarithmic negativity (figures 2, 3, 4 and 10), therefore by knowing the proper formula for describing the dynamics of entanglement entropy and logarithmic negativity, we can find a proper formula for the odd entanglement entropy. Chapman, Eisert, Hackl, Heller, Jefferson, Marrochio and Myers have provided a phenomenological formula for the entanglement entropy in a similar setup, equation (1.3). Furthermore, another phenomenological formula is proposed by Alba and Calabrese [44] for the logarithmic negativity in another similar setup (right or left side in our construction and in the decompactification limit),

$$
\begin{aligned}
\mathcal{E}=\sum_{n} \tilde{s}_{n}^{\operatorname{TFD}}\left[\operatorname{Max}\left(\frac{d}{2}, 2\left|v_{n}\right| t\right)+\right. & \operatorname{Max}\left(\frac{d+2 l_{1}+2 l_{2}}{2}, 2\left|v_{n}\right| t\right) \\
& \left.-2 \operatorname{Max}\left(\frac{d+l_{1}+l_{2}}{2}, 2\left|v_{n}\right| t\right)\right],
\end{aligned}
$$

where the velocities $v_{n}$ are same as (1.2). Albeit this formula is valid before the finite size effects to be appeared i.e. only for times $t<\mathcal{L}-\left(l_{1}+l_{2}\right)$, but one can include them by carefully tracking quasi-particles on a circle leaving and re-entering the interval. More importantly, in contrast to the expression of $s_{n}^{\text {TFD }}$ in (1.4) for the entanglement entropy, fixing $\tilde{s}_{n}^{\text {TFD }}$ for the odd entanglement entropy is not easy and to the date is the main obstacle to make (6.1) predictive. It would be very interesting to be able to find this function, since by knowing that we can calculate the logarithmic negativity and then by adding the contribution of entanglement entropy (1.3), we can find a phenomenological formula for the odd entanglement entropy. We hope to address this question in our near future work. Last but not least, it is exciting to explore the logarithmic growth at intermediate times for different measures in the holographic context.

\section{Acknowledgments}

Special thanks to Mohsen Alishahiha, Pascuale Calabrese, Lucas Fabian Hackl, Yuya Kusuki, Ali Mollabashi, Behrad Taghavi, Kotaro Tamaoka and Erik Tonni for useful comments and fruitful discussions. Authors thank Behrad Taghavi and Erik Tonni for carefully reading the draft and IPM-Grid computing group for providing computing and storage facilities. Part of MG work is supported by Iran Science Elites Federation (ISEF). Also, authors would like to thank the referee for her/his valuable comments and suggestions. 

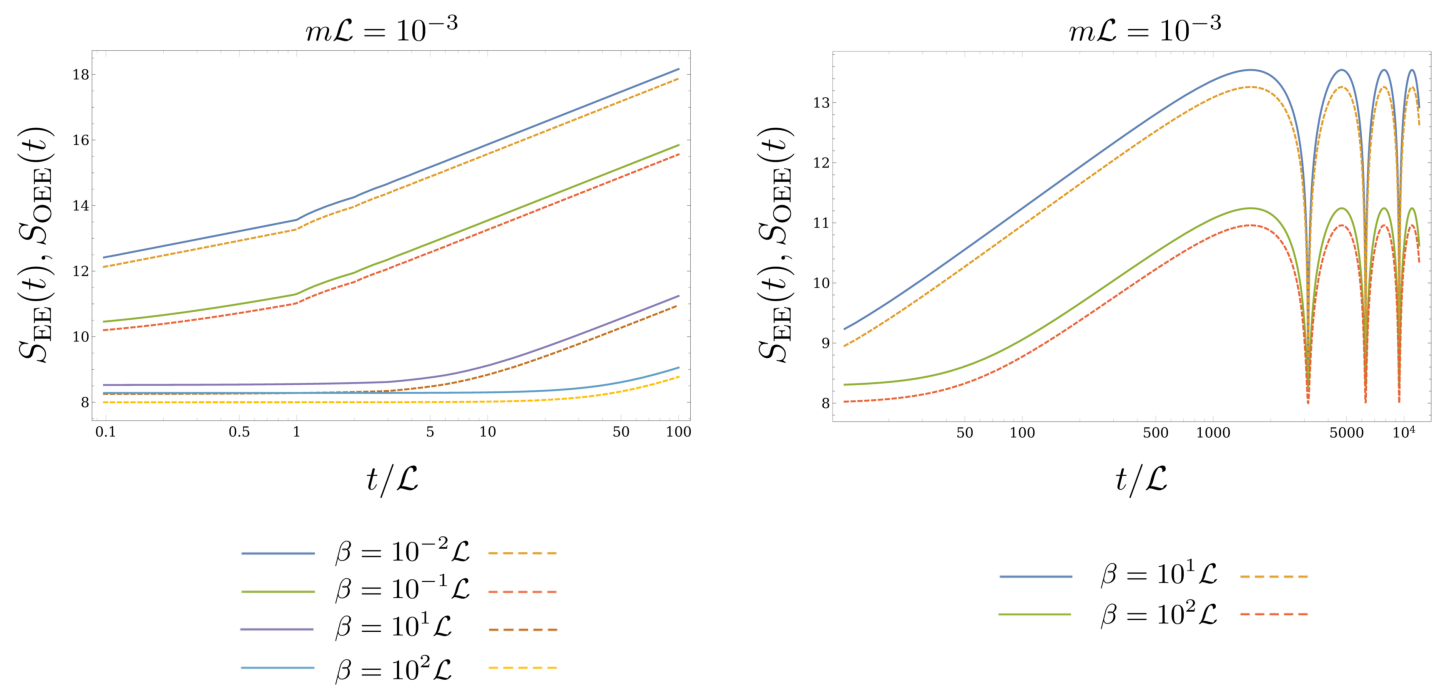

Figure 11. Temperature effect for adjacent configurations: the time dependence of $S_{\mathrm{OEE}}$ (dashed) and $S_{\mathrm{EE}}$ (solid) are presented for different temperatures. We take $N=1501, N_{A_{L(R)}}=N_{A_{L_{1}\left(R_{1}\right)}}+$ $N_{A_{L_{2}\left(R_{2}\right)}}=1+1$.

\section{A Temperature effects: various entanglement measures}

In this appendix, we consider the effect of changing temperature on $S_{\mathrm{EE}}, S_{\mathrm{OEE}}, \mathcal{E}$, and $\Delta S=S_{\mathrm{OEE}}-S_{\mathrm{EE}}$. As we will see, the behavior of OEE is same as EE and both of them approach thermal entropy by increasing temperature. This confirms that OEE is a measure of both classical and quantum correlations. However, the effect of decreasing temperature on LN behavior is only a delay in its initial growth which indicates that the LN is a measure of quantum correlation. The adjacent and disjoint subsystem configurations will be considered separately.

\section{A.1 Two adjacent intervals on each side}

In figure 11, left panel, time evolution of the $S_{\mathrm{EE}}$ and $S_{\mathrm{OEE}}$ are presented for temperatures ranging from $\beta=10^{-2} \mathcal{L}$ to $10^{2} \mathcal{L}$. In this panel, the upper two curves present hightemperature limit where the thermal correlation dominates. For low temperatures, the initial growth happens after a delay. The right panel shows the finite size effects for different temperatures. The time dependence of $\Delta S=S_{\mathrm{OEE}}-S_{\mathrm{EE}}$ is presented in figure 12, for various ranges of temperatures. The left panel shows the initial decreasing and the left one shows saturation. In initial times, the difference is bigger for high temperatures in comparison with low temperatures.

In figures 13 and 14, the time evolution of $\mathcal{E}$ is studied for temperatures ranging from $\beta=10^{-2} \mathcal{L}$ to $10^{2} \mathcal{L}$. The temperature decreases by a factor of 10 from the upper-left panel to the lower-right panel. In figure 13, the upper panels denote the time evolution for high temperatures and the bottom panels are related to low temperatures. Interestingly, the $\mathrm{LN}$ is zero for times $t<\beta / 2$ and after that, it begins to grow linearly. ${ }^{25}$ Followed by

\footnotetext{
${ }^{25}$ This delay is also reported in [69].
} 

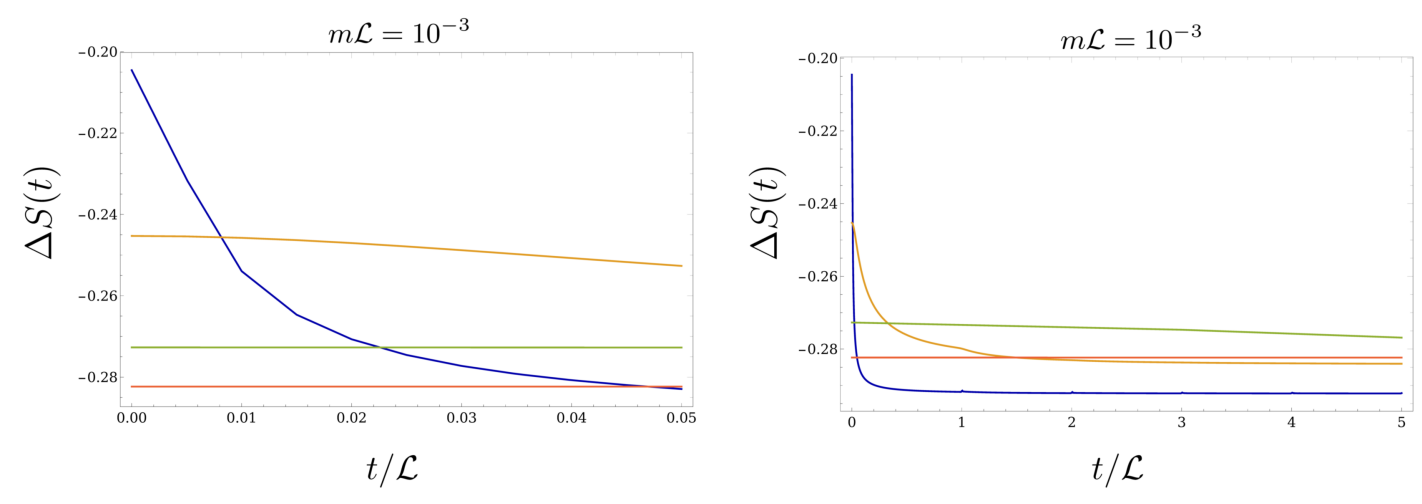

$$
\begin{aligned}
-\beta & =10^{-2} \mathcal{L} & -\beta & =10^{-1} \mathcal{L} \\
-\beta & =10^{1} \mathcal{L} & -\beta & =10^{2} \mathcal{L}
\end{aligned}
$$

Figure 12. Temperature effect on time evolution of $\Delta S=S_{\mathrm{OEE}}-S_{\mathrm{EE}}$. We take $N=1501$ and $N_{A_{L(R)}}=N_{A_{L_{1}\left(R_{1}\right)}}+N_{A_{L_{2}\left(R_{2}\right)}}=1+1$.
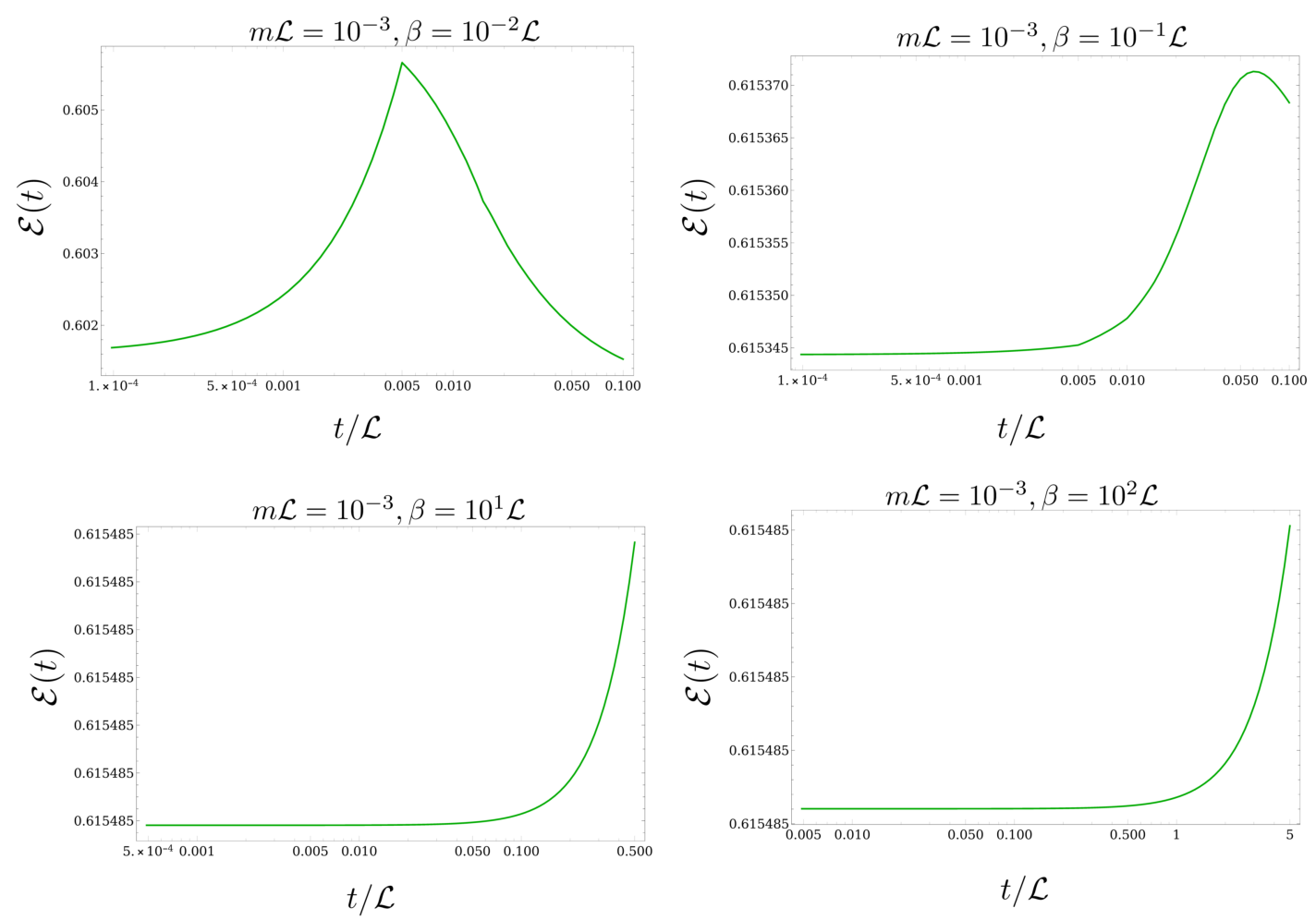

Figure 13. Temperature effect on initial growth of logarithmic negativity $\mathcal{E}$. We take $N=1501$, $N_{A_{L(R)}}=N_{A_{L_{1}\left(R_{1}\right)}}+N_{A_{L_{2}\left(R_{2}\right)}}=1+1$.

figure 13, the long time behavior of logarithmic negativity for various temperatures are presented in figure 14. From upper panels, which are correspond to high temperatures $\beta=10^{-2} \mathcal{L}$ (upper-left) and $\beta=10^{-1} \mathcal{L}$ (upper-right), we see the reduction of logarithmic negativity for time scales of order $t \sim \mathcal{L}$ and sudden quantum revival after this time. The finite size effects induce a periodic behavior. For bottom panels, which correspond to 

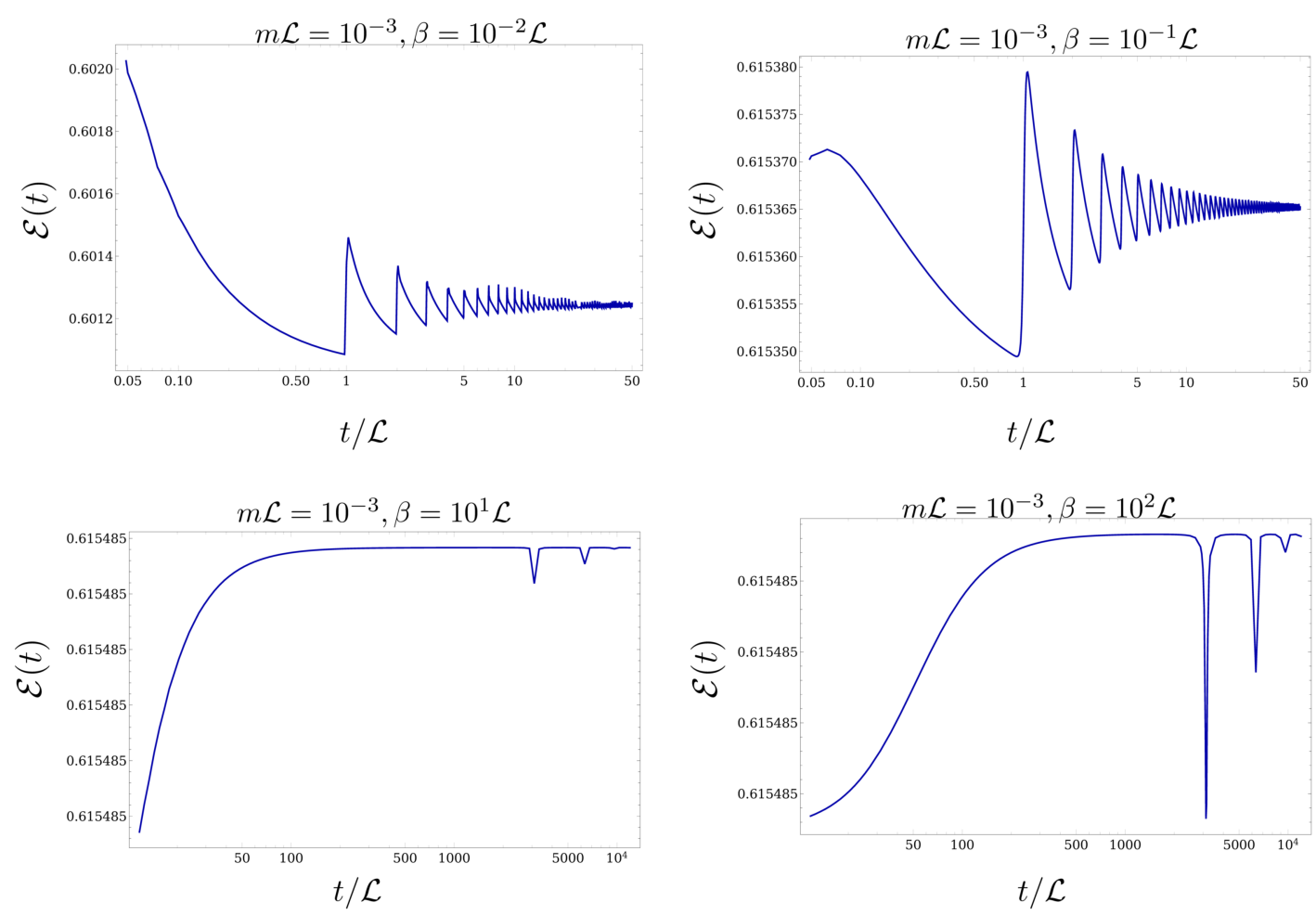

Figure 14. Temperature effect on long time behavior of logarithmic negativity $\mathcal{E}$. We take $N=1501$, $N_{A_{L(R)}}=N_{A_{L_{1}\left(R_{1}\right)}}+N_{A_{L_{2}\left(R_{2}\right)}}=1+1$.

the low temperatures, $\beta=10 \mathcal{L}$ (bottom-left) and $\beta=10^{2} \mathcal{L}$ (bottom-right), we observe the growth of logarithmic negativity followed by a plateau and then an oscillatory behavior due to the finite size effect.

\section{A.2 Two disjoint intervals on each side}

In this subsection, we will study the effect of changing separation $d$ and temperature together on the time evolution of $S_{\mathrm{EE}}, S_{\mathrm{OEE}}$ and $\Delta S=S_{\mathrm{OEE}}-S_{\mathrm{EE}}$. The figure 15 denotes the time-dependent behavior of $S_{\mathrm{EE}}$ (upper panels) and $S_{\mathrm{OEE}}$ (bottom panels). Effectively, $S_{\mathrm{OEE}}$ and $S_{\mathrm{EE}}$ behave the same and there exists a competition between increasing the distance $d$ and decreasing the temperature. By increasing the distance, $\left(S_{\mathrm{OEE}}, S_{\mathrm{EE}}\right)$ increase and by decreasing the temperature both of them decrease. Therefore, in general, it exists a critical ratio $d_{\mathrm{cr}} / \beta_{\mathrm{cr}}$ which around it increasing distance or decreasing the temperature wins the competition. Figure 16 represents the time dependence of $\Delta S=S_{\mathrm{OEE}}-S_{\mathrm{EE}}$ with initial fluctuation around zero value followed by saturation. The effects of finite size can be seen as oscillatory behavior.

\section{B Mass effects: various entanglement measures}

In this appendix, we present the time evolution of $S_{\mathrm{EE}}, S_{\mathrm{OEE}}$ and $\mathcal{E}$ in free massive scalar theory for various distances $d$ between entangling regions. In figure 17, the upper-left plot denotes the linear regime for the growth of entanglement entropy. The linear growth is for 

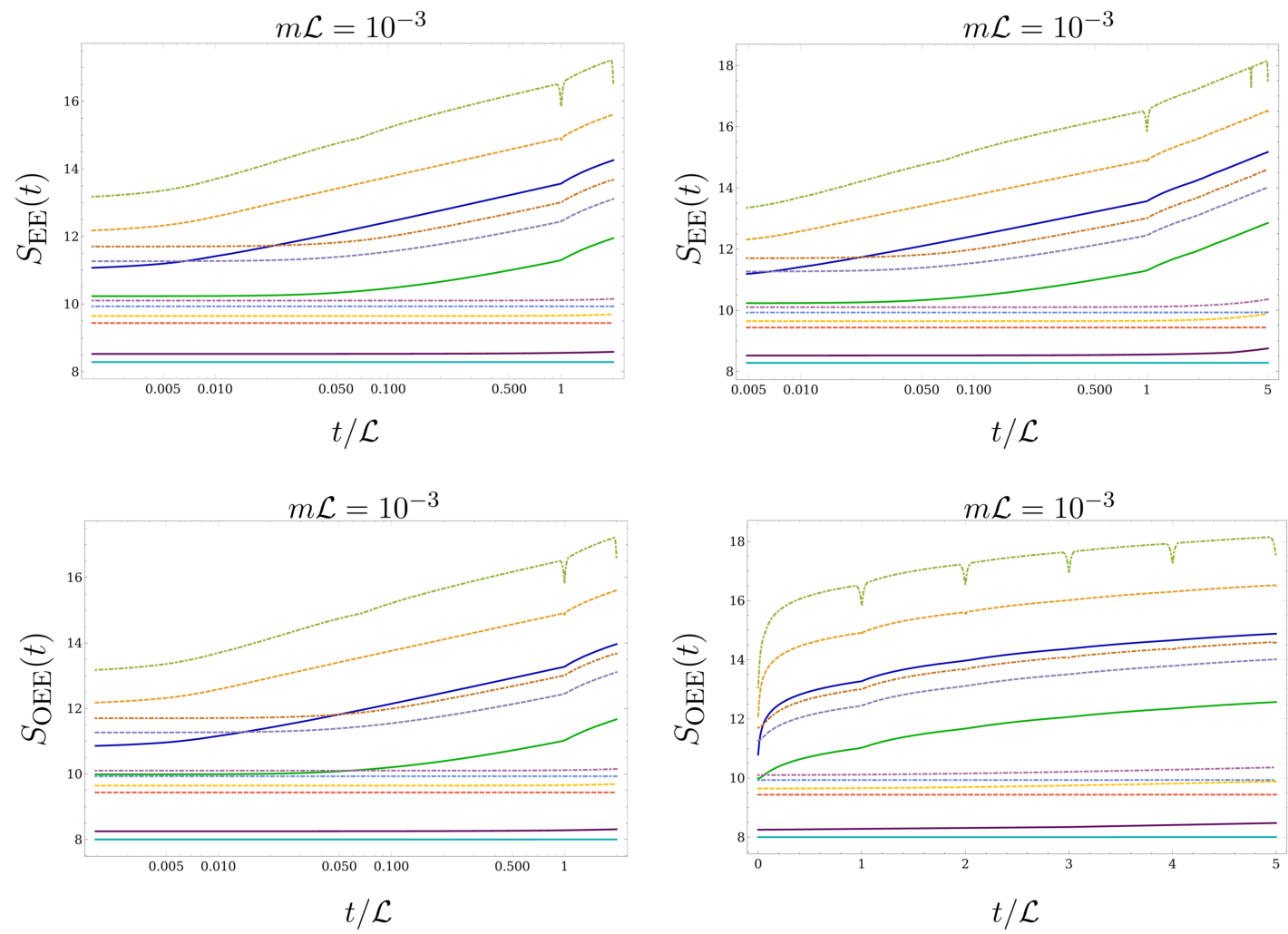

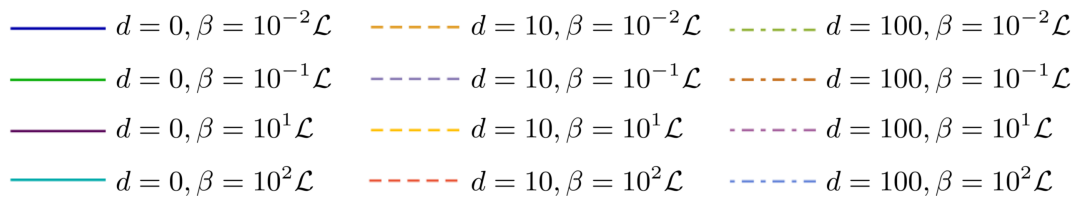

Figure 15. Temperature effect for disjoint configurations. The time evolution of $S_{\mathrm{EE}}$ and $S_{\mathrm{OEE}}$ are presented for various temperatures and various subregion distances. We take $N=1501, N_{A_{L(R)}}=$ $N_{A_{L_{1}\left(R_{1}\right)}}+N_{A_{L_{2}\left(R_{2}\right)}}=1+1$.

times approximately equal to the size of an interval. The upper-right plot is for a longer period of time for which the finite size effects induce a periodic behavior with periodicity $\mathcal{L}$. The lower-left and right panels denote the time behavior of odd entanglement entropy. The effective evolution of $S_{\mathrm{EE}}$ and $S_{\mathrm{OEE}}$ are the same in different times. Moreover, it is clear that for large masses, the oscillations are rather irregular due to the dephasing of the different kinds of quasi-particles with different group velocities. By increasing the separation distance $d$, we also observe the memory effect [77] in $S_{\mathrm{EE}}$ and $S_{\mathrm{OEE}}$ when $d>l_{1}+$ $l_{2}$. Furthermore, the effect of changing the mass on the time evolution of $\Delta S=S_{\mathrm{OEE}}-S_{\mathrm{EE}}$ and $\mathcal{E}$ is provided in figure 18. Apart from the appearance of irregular oscillations, the time evolution for both of them is the same and it is the sign that they are actually proportional together. 

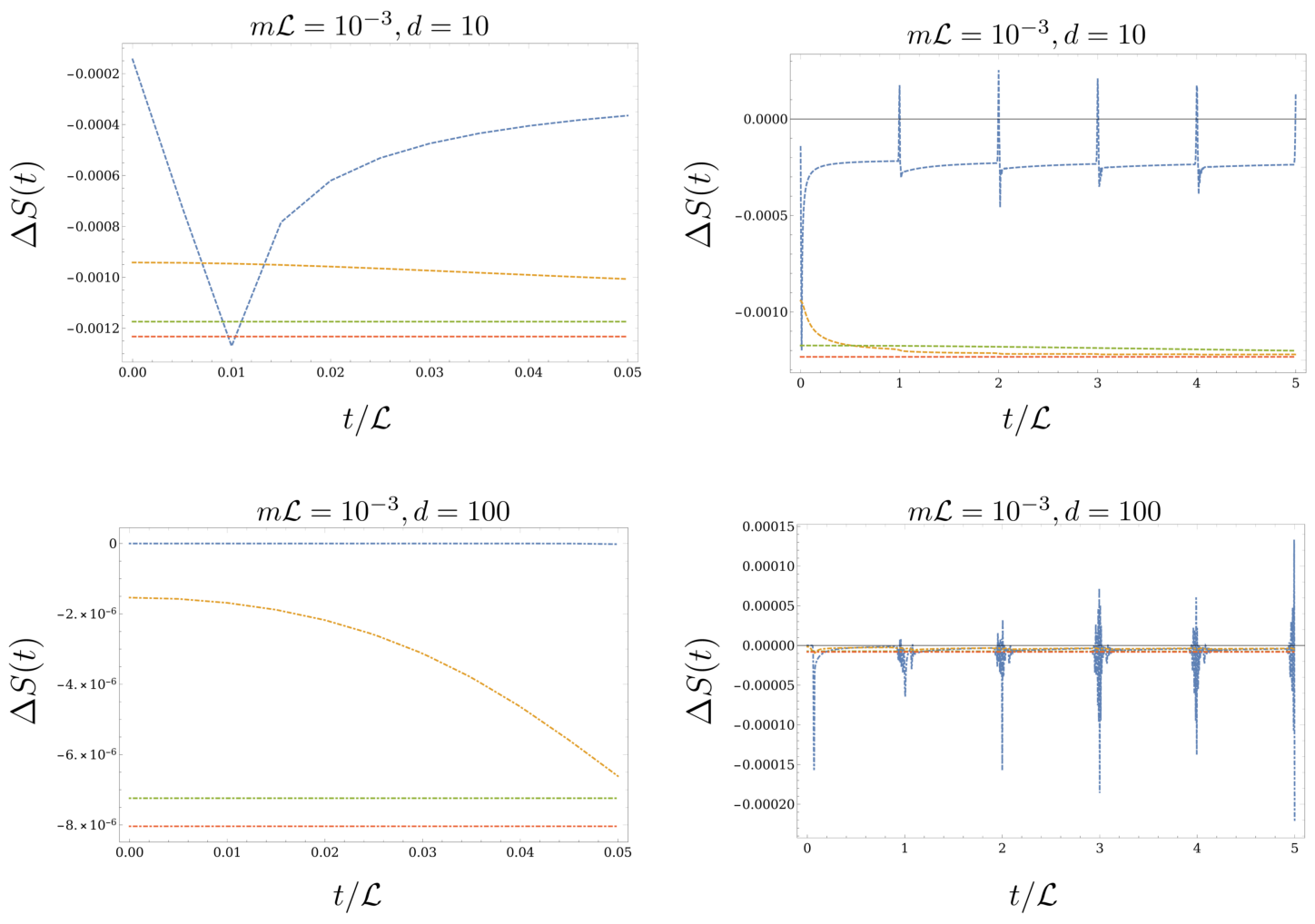

$$
\begin{aligned}
& ----\beta=10^{-2} \mathcal{L} \\
& ----\beta=10^{1} \mathcal{L}
\end{aligned}
$$

$$
\begin{aligned}
----\beta & =10^{-1} \mathcal{L} \\
----\beta & =10^{2} \mathcal{L}
\end{aligned}
$$

Figure 16. Temperature effect on time evolution of $\Delta S=S_{\mathrm{OEE}}-S_{\mathrm{EE}}$ is presented for $N=1501$, $N_{A_{L(R)}}=N_{A_{L_{1}\left(R_{1}\right)}}+N_{A_{L_{2}\left(R_{2}\right)}}=1+1$.

\section{Zero-mode computations}

In this appendix, we provide details of calculations in section 5. Using (5.9), the related covariance matrix in the position basis $\xi_{x}^{a}=\left(q_{x}^{L}, q_{x}^{R}, p_{x}^{L}, p_{x}^{R}\right)$ takes the following form:

$$
\begin{aligned}
G_{x, x}^{a b}(t)= & \frac{1}{N} \sum_{k}^{N-1} \\
& \times\left(\begin{array}{cccc}
\frac{\cosh \left(2 \alpha_{k}\right)}{\lambda_{k}} & \frac{\cos \left(\omega_{k} t\right) \sinh \left(2 \alpha_{k}\right)}{\lambda_{k}} & 0 & -\sin \left(\omega_{k} t\right) \sinh \left(2 \alpha_{k}\right) \\
\frac{\cos \left(\omega_{k} t\right) \sinh \left(2 \alpha_{k}\right)}{\lambda_{k}} & \frac{\cosh \left(2 \alpha_{k}\right)}{\lambda_{k}} & -\sin \left(\omega_{k} t\right) \sinh \left(2 \alpha_{k}\right) & 0 \\
0 & -\sin \left(\omega_{k} t\right) \sinh \left(2 \alpha_{k}\right) & \lambda_{k} \cosh \left(2 \alpha_{k}\right) & -\lambda_{k} \cos \left(\omega_{k} t\right) \sinh \left(2 \alpha_{k}\right) \\
-\sin \left(\omega_{k} t\right) \sinh \left(2 \alpha_{k}\right) & 0 & -\lambda_{k} \cos \left(\omega_{k} t\right) \sinh \left(2 \alpha_{k}\right) & \lambda_{k} \cosh \left(2 \alpha_{k}\right)
\end{array}\right) .
\end{aligned}
$$

The schematic form of this matrix is

$$
G_{x, x}^{a b}(t)=\left(\begin{array}{cccc}
e_{1} & \mathcal{M}_{1}(t) & 0 & \mathcal{M}_{2}(t) \\
\mathcal{M}_{1}(t) & e_{1} & \mathcal{M}_{2}(t) & 0 \\
0 & \mathcal{M}_{2}(t) & e_{2} & \mathcal{M}_{3}(t) \\
\mathcal{M}_{2}(t) & 0 & \mathcal{M}_{3}(t) & e_{2}
\end{array}\right)
$$



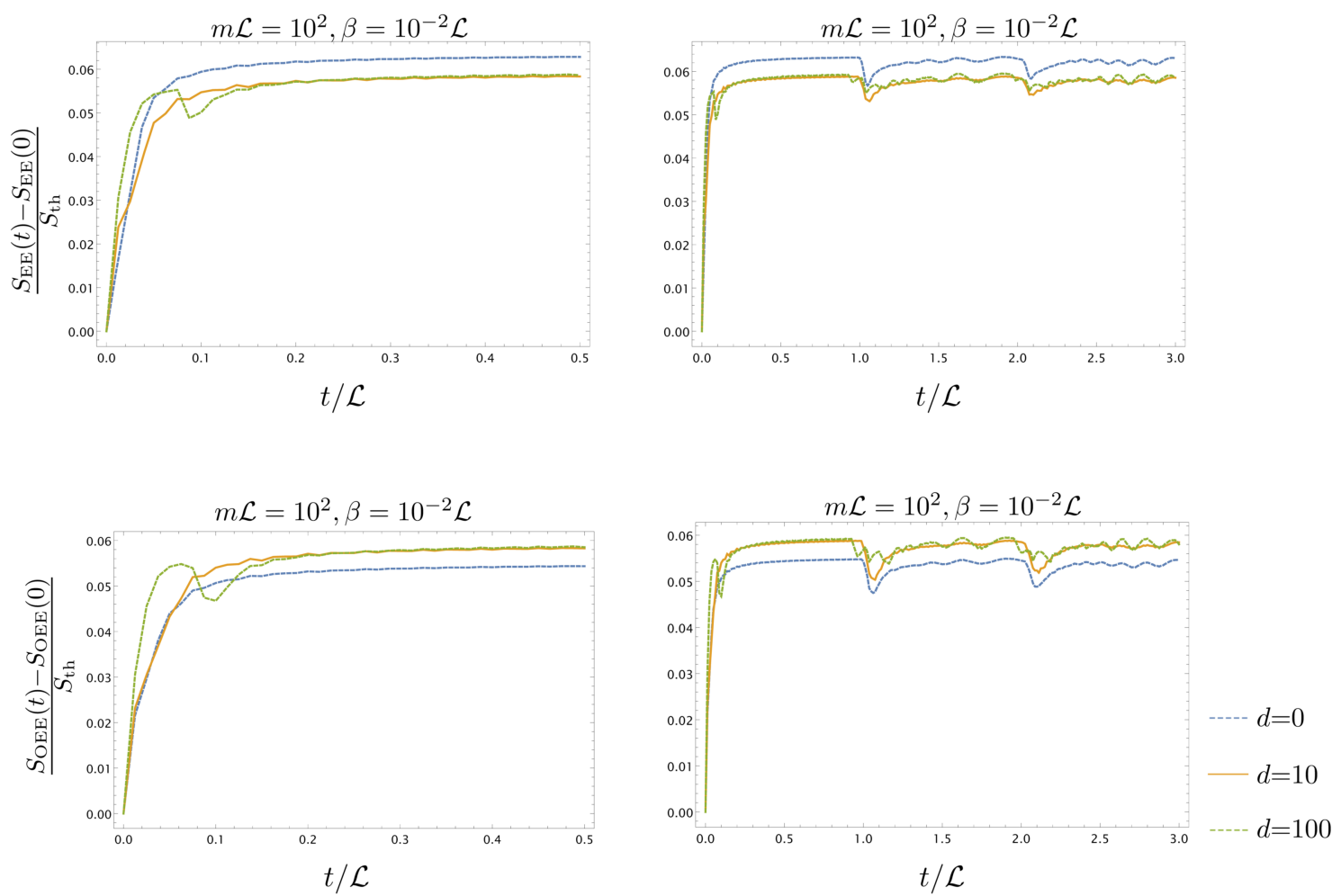

Figure 17. Mass effect on time evolution of $S_{\mathrm{EE}}$ (upper panels) and $S_{\mathrm{OEE}}$ (bottom panels). We take $N=1501$ and $N_{A_{L(R)}}=N_{A_{L_{1}\left(R_{1}\right)}}+N_{A_{L_{2}\left(R_{2}\right)}}=20+31$.
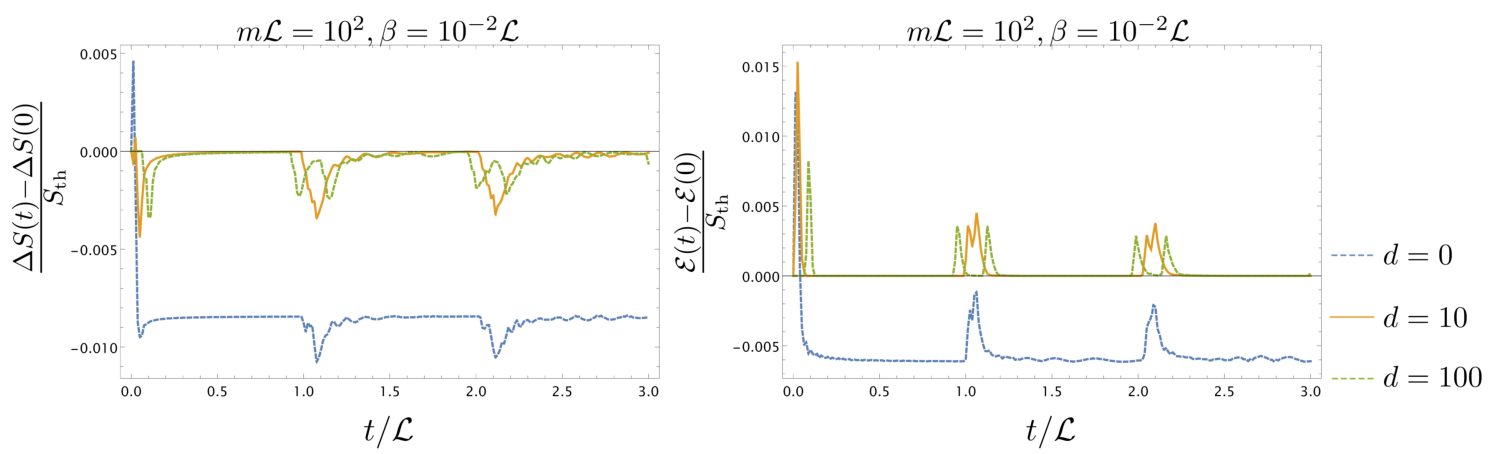

Figure 18. Mass effect on long-time evolution of $\Delta S=S_{\mathrm{OEE}}-S_{\mathrm{EE}}$ and $\mathcal{E}$. These are normalized by the thermodynamic entropy $S_{\text {th }}$ when $N=1501, N_{A_{L(R)}}=N_{A_{L_{1}\left(R_{1}\right)}}+N_{A_{L_{2}\left(R_{2}\right)}}=20+31$.

whose determinant becomes

$$
\operatorname{det}\left(G_{x, x}^{a b}(t)\right)=\underbrace{e_{1}^{2} e_{2}^{2}-e_{2}^{2} F_{1}^{2}(t)}_{\text {Leading order }}+\underbrace{\left(\mathcal{M}_{2}^{2}(t)-\mathcal{M}_{1}(t) \mathcal{M}_{2}(t)\right)^{2}-2 e_{1} e_{2} \mathcal{M}_{2}^{2}(t)-e_{1}^{2} \mathcal{M}_{3}^{2}(t)}_{\text {Subleading order }} \text {. }
$$

When $t \gg \delta=\frac{\mathcal{L}}{N}$ and $N \rightarrow \infty, m \mathcal{L} \ll 1, \beta / \mathcal{L} \ll 1$ and $m \ll \delta^{-1}$, one can see that

$$
\begin{aligned}
\sinh \left(2 \alpha_{k}\right) & =1 / \sinh \left(\frac{\beta \omega_{k}}{2}\right)_{k \ll N, \beta \ll \mathcal{L}} \frac{2}{\beta \omega_{k}} \\
\omega_{k} & =\omega_{N-k} \sim \frac{2 \pi k}{\mathcal{L}}
\end{aligned}
$$


which altogether imply that

$$
\begin{aligned}
e_{1} & =\frac{1}{N} \sum_{k=0}^{N-1} \frac{\cosh \left(2 \alpha_{k}\right)}{\lambda_{k}} \\
& =\frac{1}{N}\left(\frac{\cosh \left(2 \alpha_{0}\right)}{\lambda_{0}}+\sum_{k=1}^{N-1} \frac{\cosh \left(2 \alpha_{k}\right)}{\lambda_{k}}\right) \\
& =\frac{1}{N}\left(\frac{\cosh \left(2 \alpha_{0}\right)}{\lambda_{0}}+2 \sum_{k=1}^{\frac{N}{2}} \frac{\cosh \left(2 \alpha_{k}\right)}{\lambda_{k}}\right) \\
& =\frac{2}{N \beta \mathcal{L} m^{2}}\left(1+\frac{m^{2} \mathcal{L}^{2}}{2 \pi^{2}} \sum_{k=1}^{\infty} \frac{1}{k^{2}}\right) \sim \frac{2}{N \beta \mathcal{L} m^{2}}\left(1+\frac{m^{2} \mathcal{L}^{2}}{12}\right), \\
e_{2} & =\frac{1}{N} \sum_{k=0}^{N-1} \lambda_{k} \cosh \left(2 \alpha_{k}\right) \\
& =\frac{1}{N}\left(\lambda_{0} \cosh \left(2 \alpha_{0}\right)+\sum_{k=1}^{N-1} \lambda_{k} \cosh \left(2 \alpha_{k}\right)\right) \\
& =\frac{1}{N}\left(\frac{2 \mathcal{L}}{\beta}+2 \sum_{k=1}^{\frac{N}{2}} \frac{2 \mathcal{L}}{\beta}\right) \sim \frac{2 \mathcal{L}}{\beta},
\end{aligned}
$$

and

$$
\begin{aligned}
\mathcal{M}_{1}(t) & =\frac{1}{N} \sum_{k=0}^{N-1} \frac{\cos \left(\omega_{k} t\right) \sinh \left(2 \alpha_{k}\right)}{\lambda_{k}} \\
& =\frac{1}{N}\left(\frac{\cos \left(\omega_{0} t\right) \sinh \left(2 \alpha_{0}\right)}{\lambda_{0}}+\sum_{k=1}^{N-1} \frac{\cos \left(\omega_{k} t\right) \sinh \left(2 \alpha_{k}\right)}{\lambda_{k}}\right) \\
& =\frac{1}{N}\left(\frac{\cos \left(\omega_{0} t\right) \sinh \left(2 \alpha_{0}\right)}{\lambda_{0}}+2 \sum_{k=1}^{\frac{N}{2}} \frac{\cos \left(\omega_{k} t\right) \sinh \left(2 \alpha_{k}\right)}{\lambda_{k}}\right) \\
& \sim \frac{2}{N \beta \mathcal{L} m}\left(\frac{\cos \left(\omega_{0} t\right)}{m}+\frac{m \mathcal{L}^{2}}{2 \pi^{2}} \sum_{k=1}^{\infty} \frac{\cos \left(\omega_{k} t\right)}{k^{2}}\right) \\
& \sim \frac{2}{N \beta \mathcal{L} m}\left(\frac{\cos (m t)}{m}+\frac{m \mathcal{L}^{2}}{2 \pi^{2}} \sum_{k=1}^{\infty} \frac{\cos \left(\omega_{k} t\right)}{k^{2}}\right), \\
\mathcal{M}_{2}(t) & =\frac{-1}{N} \sum_{k=0}^{N-1} \sin \left(\omega_{k} t\right) \sinh \left(2 \alpha_{k}\right) \\
& =\frac{-1}{N}\left(\sin \left(\omega_{0} t\right) \sinh \left(2 \alpha_{0}\right)+\sum_{k=1}^{N-1} \sin \left(\omega_{k} t\right) \sinh \left(2 \alpha_{k}\right)\right) \\
& =\frac{-1}{N}\left(\sin \left(\omega_{0} t\right) \sinh \left(2 \alpha_{0}\right)+2 \sum_{k=1}^{\frac{N}{2}} \sin \left(\omega_{k} t\right) \sinh \left(2 \alpha_{k}\right)\right) \\
& \sim \frac{-2}{N \beta}\left(\frac{\sin \left(\omega_{0} t\right)}{m}+\frac{\mathcal{L}}{\pi} \sum_{k=1}^{\infty} \frac{\sin \left(\omega_{k} t\right)}{k}\right) \\
& -2\left(\frac{\sin (m t)}{m}+\frac{\mathcal{L}}{\pi} \sum_{k=1}^{\infty} \frac{\sin \left(\omega_{k} t\right)}{k}\right), \\
k &
\end{aligned}
$$




$$
\begin{aligned}
\mathcal{M}_{3}(t) & =\frac{-1}{N} \sum_{k}^{N-1} \lambda_{k} \cos \left(\omega_{k} t\right) \sinh \left(2 \alpha_{k}\right) \\
& =\frac{-1}{N}\left(\lambda_{0} \cos \left(\omega_{0} t\right) \sinh \left(2 \alpha_{0}\right)+\sum_{k=1}^{N-1} \lambda_{k} \cos \left(\omega_{k} t\right) \sinh \left(2 \alpha_{k}\right)\right) \\
& =\frac{-1}{N}\left(\lambda_{0} \cos \left(\omega_{0} t\right) \sinh \left(2 \alpha_{0}\right)+2 \sum_{k=1}^{\frac{N}{2}} \lambda_{k} \cos \left(\omega_{k} t\right) \sinh \left(2 \alpha_{k}\right)\right) \\
& \sim \frac{-2 \mathcal{L} m}{N \beta}\left(\frac{\cos \left(\omega_{0} t\right)}{m}+\frac{2}{m} \sum_{k=1}^{\infty} \frac{k \cos \left(\omega_{k} t\right)}{k}\right) \\
& \sim \frac{-2 \mathcal{L} m}{N \beta}\left(\frac{\cos \left(\omega_{0} t\right)}{m}+\frac{2}{m} \sum_{k=1}^{\infty} \cos \left(\omega_{k} t\right)\right)
\end{aligned}
$$

According to the above asymptotic results, the leading order contribution for $\operatorname{det}\left(G_{x, x}^{a b}(t)\right)$ in (C.3) is given by

$$
\begin{aligned}
e_{1}^{2} e_{2}^{2}-e_{2}^{2} \mathcal{M}_{1}^{2}(t) & =\frac{4 e_{2}^{2}}{N^{2} \beta^{2} m^{4} \mathcal{L}^{2}}\left[\left(1+\frac{m^{2} \mathcal{L}^{2}}{2 \pi^{2}} \sum_{k=1}^{\infty} \frac{1}{k^{2}}\right)^{2}+\left(\cos (m t)+\frac{m^{2} \mathcal{L}^{2}}{2 \pi^{2}} \sum_{k=1}^{\infty} \frac{\cos \left(\omega_{k} t\right)}{k^{2}}\right)^{2}\right] \\
& =\frac{4 e_{2}^{2}}{N^{2} \beta^{2} m^{2}(m)^{2} \mathcal{L}^{2}}\left[\sin ^{2}(m t)+\frac{m^{2} \mathcal{L}^{2}}{\pi^{2}} \sum_{k=1}^{\infty} \frac{1-\cos \left[\left(\frac{2 \pi k}{\mathcal{L}}\right) t\right]}{k^{2}}\right] \\
& \left.=\frac{4 e_{2}^{2}}{N^{2} \beta^{2} m^{2}}\left[\frac{\sin ^{2}(m t)+Q}{m^{2} \mathcal{L}^{2}}\right], \quad \text { (C. } 10\right)
\end{aligned}
$$

where we have defined $Q$ as

$$
Q=\frac{m^{2} \mathcal{L}^{2}}{\pi^{2}} \sum_{k=1}^{\infty} \frac{1-\cos \left[\left(\frac{2 \pi k}{\mathcal{L}}\right) t\right]}{k^{2}}
$$

Therefore, (5.2) implies that

$$
S_{\mathrm{OEE}}\left(\rho_{A}(t)\right) \sim 2(1-2 \log 2)+\log \left(\frac{2 e_{2}}{N \beta m}\right)+\frac{1}{2} \log \left[\frac{\sin ^{2}[m t]+Q}{m^{2} \mathcal{L}^{2}}\right] .
$$

Open Access. This article is distributed under the terms of the Creative Commons Attribution License (CC-BY 4.0), which permits any use, distribution and reproduction in any medium, provided the original author(s) and source are credited.

\section{References}

[1] A. Polkovnikov, K. Sengupta, A. Silva and M. Vengalattore, Nonequilibrium dynamics of closed interacting quantum systems, Rev. Mod. Phys. 83 (2011) 863 [arXiv:1007.5331] [INSPIRE].

[2] A.M. Kaufman et al., Quantum thermalization through entanglement in an isolated many-body system, Science 353 (2016) 794 [arXiv:1603.04409]. 
[3] C. Gogolin and J. Eisert, Equilibration, thermalisation, and the emergence of statistical mechanics in closed quantum systems, Rept. Prog. Phys. 79 (2016) 056001 [arXiv: 1503.07538] [INSPIRE].

[4] F.H.L. Essler and M. Fagotti, Quench dynamics and relaxation in isolated integrable quantum spin chains, J. Stat. Mech. 1606 (2016) 064002 [arXiv:1603.06452] [INSPIRE].

[5] L. Vidmar and M. Rigol, Generalized Gibbs ensemble in integrable lattice models, J. Stat. Mech. 06 (2016) 064007 [arXiv: 1604.03990].

[6] P. Calabrese and J.L. Cardy, Evolution of entanglement entropy in one-dimensional systems, J. Stat. Mech. 0504 (2005) P04010 [cond-mat/0503393] [INSPIRE].

[7] P. Calabrese and J.L. Cardy, Time-dependence of correlation functions following a quantum quench, Phys. Rev. Lett. 96 (2006) 136801 [cond-mat/0601225] [INSPIRE].

[8] P. Calabrese and J. Cardy, Quantum quenches in extended systems, J. Stat. Mech. 0706 (2007) P06008 [arXiv:0704.1880] [INSPIRE].

[9] V. Alba and P. Calabrese, Entanglement and thermodynamics after a quantum quench in integrable systems, PNAS 114 (2017) 7947 [arXiv:1608.00614v2].

[10] V. Alba and P. Calabrese, Entanglement dynamics after quantum quenches in generic integrable systems, SciPost Phys. 4 (2018) 017 [arXiv:1712.07529].

[11] R. Horodecki, P. Horodecki, M. Horodecki and K. Horodecki, Quantum entanglement, Rev. Mod. Phys. 81 (2009) 865 [quant-ph/0702225] [INSPIRE].

[12] E.H. Lieb, D.W. Robinson, The finite group velocity of quantum spin systems, Commun. Math. Phys. 28 (1972) 251.

[13] S. Chapman et al., Complexity and entanglement for thermofield double states, SciPost Phys. 6 (2019) 034 [arXiv: 1810.05151] [INSPIRE].

[14] M.B. Plenio and S. Virmani, An introduction to entanglement measures, Quant. Inf. Comput. 7 (2007) 1 [quant-ph/0504163] [INSPIRE].

[15] J. Eisert and M.B. Plenio, A comparison of entanglement measures, J. Mod. Opt. 46 (1999) 145 [quant-ph/9807034] [INSPIRE].

[16] G. Vidal and R.F. Werner, Computable measure of entanglement, Phys. Rev. A 65 (2002) 032314 [quant-ph/0102117] [INSPIRE].

[17] M.B. Plenio, Logarithmic negativity: a full entanglement monotone that is not convex, Phys. Rev. Lett. 95 (2005) 090503 [quant-ph/0505071] [INSPIRE].

[18] K. Tamaoka, Entanglement wedge cross section from the dual density matrix, Phys. Rev. Lett. 122 (2019) 141601 [arXiv: 1809.09109] [INSPIRE].

[19] O. Aharony, S.S. Gubser, J.M. Maldacena, H. Ooguri and Y. Oz, Large N field theories, string theory and gravity, Phys. Rept. 323 (2000) 183.

[20] J. Kudler-Flam and S. Ryu, Entanglement negativity and minimal entanglement wedge cross sections in holographic theories, Phys. Rev. D 99 (2019) 106014 [arXiv:1808.00446] [INSPIRE].

[21] Y. Kusuki, J. Kudler-Flam and S. Ryu, Derivation of holographic negativity in $A d S_{3} / \mathrm{CFT}_{2}$, Phys. Rev. Lett. 123 (2019) 131603 [arXiv: 1907.07824] [INSPIRE]. 
[22] T. Takayanagi and K. Umemoto, Entanglement of purification through holographic duality, Nature Phys. 14 (2018) 573 [arXiv:1708.09393].

[23] P. Nguyen, T. Devakul, M.G. Halbasch, M.P. Zaletel and B. Swingle, Entanglement of purification: from spin chains to holography, JHEP 01 (2018) 098 [arXiv:1709.07424] [INSPIRE].

[24] Y. Kusuki and K. Tamaoka, Dynamics of entanglement wedge cross section from conformal field theories, Phys. Lett. B $\mathbf{8 1 4}$ (2021) 136105 [arXiv:1907.06646] [INSPIRE].

[25] Y. Kusuki and K. Tamaoka, Entanglement wedge cross section from CFT: dynamics of local operator quench, JHEP 02 (2020) 017 [arXiv: 1909.06790] [INSPIRE].

[26] K. Babaei Velni, M.R. Mohammadi Mozaffar and M.H. Vahidinia, Evolution of entanglement wedge cross section following a global quench, JHEP 08 (2020) 129 [arXiv:2005. 05673] [INSPIRE].

[27] M. Sahraei, M.J. Vasli, M.R.M. Mozaffar and K.B. Velni, Entanglement wedge cross section in holographic excited states, arXiv:2105.12476 [INSPIRE].

[28] J. Kudler-Flam, Y. Kusuki and S. Ryu, Correlation measures and the entanglement wedge cross-section after quantum quenches in two-dimensional conformal field theories, JHEP 04 (2020) 074 [arXiv : 2001.05501] [inSPIRE].

[29] J. Kudler-Flam, Y. Kusuki and S. Ryu, The quasi-particle picture and its breakdown after local quenches: mutual information, negativity, and reflected entropy, JHEP 03 (2021) 146 [arXiv: 2008.11266] [INSPIRE].

[30] X. Dong, X.-L. Qi and M. Walter, Holographic entanglement negativity and replica symmetry breaking, JHEP 06 (2021) 024 [arXiv:2101.11029] [INSPIRE].

[31] A. Mollabashi and K. Tamaoka, A field theory study of entanglement wedge cross section: odd entropy, JHEP 08 (2020) 078 [arXiv: 2004.04163] [INSPIRE].

[32] A. Peres, Separability criterion for density matrices, Phys. Rev. Lett. 77 (1996) 1413 [quant-ph/9604005] [INSPIRE].

[33] M. Horodecki, P. Horodecki and R. Horodecki, On the necessary and sufficient conditions for separability of mixed quantum states, Phys. Lett. A 223 (1996) 1 [quant-ph/9605038] [INSPIRE].

[34] R. Simon, Peres-Horodecki separability criterion for continuous variable systems, Phys. Rev. Lett. 84 (2000) 2726 [quant-ph/9909044] [INSPIRE].

[35] K. Audenaert, J. Eisert, M.B. Plenio and R.F. Werner, Entanglement properties of the harmonic chain, Phys. Rev. A 66 (2002) 042327 [quant-ph/0205025] [INSPIRE].

[36] P. Calabrese, J. Cardy and E. Tonni, Entanglement negativity in quantum field theory, Phys. Rev. Lett. 109 (2012) 130502 [arXiv:1206.3092] [INSPIRE].

[37] P. Calabrese, J. Cardy and E. Tonni, Entanglement negativity in extended systems: A field theoretical approach, J. Stat. Mech. 1302 (2013) P02008 [arXiv: 1210.5359] [INSPIRE].

[38] C. De Nobili, A. Coser and E. Tonni, Entanglement entropy and negativity of disjoint intervals in CFT: Some numerical extrapolations, J. Stat. Mech. 1506 (2015) P06021 [arXiv: 1501.04311] [INSPIRE].

[39] V. Eisler and Z. Zimboras, Entanglement negativity in two-dimensional free lattice models, Phys. Rev. B 93 (2016) 115148 [arXiv:1511.08819v2]. 
[40] P. Calabrese, J. Cardy and E. Tonni, Finite temperature entanglement negativity in conformal field theory, J. Phys. A 48 (2015) 015006 [arXiv: 1408.3043] [InSPIRE].

[41] M.R. Mohammadi Mozaffar and A. Mollabashi, Logarithmic Negativity in Lifshitz Harmonic Models, J. Stat. Mech. 1805 (2018) 053113 [arXiv:1712.03731] [INSPIRE].

[42] H. Shapourian and S. Ryu, Finite-temperature entanglement negativity of free fermions, J. Stat. Mech. 1904 (2019) 043106 [arXiv: 1807.09808] [INSPIRE].

[43] J. Angel-Ramelli, C. Berthiere, V.G.M. Puletti and L. Thorlacius, Logarithmic negativity in quantum Lifshitz theories, JHEP 09 (2020) 011 [arXiv:2002.05713] [INSPIRE].

[44] V. Alba and P. Calabrese, Quantum information dynamics in multipartite integrable systems, EPL 126 (2019) 60001 [arXiv: 1809.09119] [INSPIRE].

[45] V. Balasubramanian et al., Thermalization of strongly coupled field theories, Phys. Rev. Lett. 106 (2011) 191601 [arXiv: 1012.4753] [INSPIRE].

[46] V. Balasubramanian et al., Holographic thermalization, Phys. Rev. D 84 (2011) 026010 [arXiv: 1103.2683] [INSPIRE].

[47] H. Liu and S.J. Suh, Entanglement tsunami: universal scaling in holographic thermalization, Phys. Rev. Lett. 112 (2014) 011601 [arXiv: 1305.7244] [INSPIRE].

[48] J.M. Maldacena, Eternal black holes in Anti-de Sitter, JHEP 04 (2003) 021 [hep-th/0106112] [INSPIRE].

[49] S.H. Shenker and D. Stanford, Black holes and the butterfly effect, JHEP 03 (2014) 067 [arXiv:1306.0622] [INSPIRE].

[50] S.H. Shenker and D. Stanford, Multiple shocks, JHEP 12 (2014) 046 [arXiv:1312.3296] [INSPIRE].

[51] D.A. Roberts, D. Stanford and L. Susskind, Localized shocks, JHEP 03 (2015) 051 [arXiv: 1409.8180] [INSPIRE].

[52] T. Hartman and J. Maldacena, Time evolution of entanglement entropy from black hole interiors, JHEP 05 (2013) 014 [arXiv:1303.1080] [INSPIRE].

[53] L. Susskind, ER=EPR, GHZ, and the consistency of quantum measurements, Fortsch. Phys. 64 (2016) 72 [arXiv:1412.8483] [InSPIRE].

[54] R. Khan, C. Krishnan and S. Sharma, Circuit complexity in fermionic field theory, Phys. Rev. D 98 (2018) 126001 [arXiv: 1801.07620] [INSPIRE].

[55] S. Chapman and H.Z. Chen, Charged complexity and the thermofield double state, JHEP 02 (2021) 187 [arXiv: 1910.07508] [INSPIRE].

[56] M. Doroudiani, A. Naseh and R. Pirmoradian, Complexity for Charged Thermofield Double States, JHEP 01 (2020) 120 [arXiv:1910.08806] [INSPIRE].

[57] E. Bianchi, L. Hackl and N. Yokomizo, Linear growth of the entanglement entropy and the Kolmogorov-Sinai rate, JHEP 03 (2018) 025 [arXiv: 1709.00427] [INSPIRE].

[58] J.S. Cotler, M.P. Hertzberg, M. Mezei and M.T. Mueller, Entanglement growth after a global quench in free scalar field theory, JHEP 11 (2016) 166 [arXiv:1609.00872] [INSPIRE].

[59] L. Hackl, E. Bianchi, R. Modak and M. Rigol, Entanglement production in bosonic systems: linear and logarithmic growth, Phys. Rev. A 97 (2018) 032321 [arXiv:1710.04279] [INSPIRE]. 
[60] L. Vidmar, L. Hackl, E. Bianchi and M. Rigol, Entanglement entropy of eigenstates of quadratic fermionic hamiltonians, Phys. Rev. Lett. 119 (2017) 020601 [arXiv:1703.02979] [INSPIRE].

[61] L.F. Hackl, Aspects of Gaussian states: entanglement, squeezing and complexity, Ph.D. thesis, Pennsylvania State University, U.S.A. (2018).

[62] G. Di Giulio and E. Tonni, Complexity of mixed Gaussian states from Fisher information geometry, JHEP 12 (2020) 101 [arXiv:2006.00921] [INSPIRE].

[63] F.C. Khanna, A.P.C. Malbouisson, J.M.C. Malbouisson and A.R. Santana, Thermal quantum field theory: algebraic aspects and applications, World Scientific, Singapore (2009).

[64] A.B. Klimov and S.M. Chumakov, A group-theoretical approach to quantum optics: models of atom-field interactions, Wiley-VCH, U.S.A. (2009).

[65] R. Jefferson and R.C. Myers, Circuit complexity in quantum field theory, JHEP 10 (2017) 107 [arXiv: 1707.08570] [INSPIRE].

[66] J. Eisert and M.B. Plenio, Introduction to the basics of entanglement theory in continuous-variable systems, Int. J. Quant. Inf. 1 (2003) 479 [quant-ph/0312071].

[67] C. Weedbrook et al., Gaussian quantum information, Rev. Mod. Phys. 84 (2012) 621.

[68] L.F. Hackl, Notes on entanglemnt production in the thermal field double, unpublished.

[69] A. Coser, E. Tonni and P. Calabrese, Entanglement negativity after a global quantum quench, J. Stat. Mech. 1412 (2014) P12017 [arXiv:1410.0900] [inSPIRE].

[70] V. Eisler and Z. Zimboras, Entanglement negativity in the harmonic chain out of equilibrium, New J. Phys. 16 (2014) 123020 [arXiv:1406.5474v3].

[71] M. Hoogeveen and B. Doyon, Entanglement negativity and entropy in non-equilibrium conformal field theory, Nucl. Phys. B 898 (2015) 78 [arXiv:1412.7568] [INSPIRE].

[72] X. Wen, P.-Y. Chang and S. Ryu, Entanglement negativity after a local quantum quench in conformal field theories, Phys. Rev. B 92 (2015) 075109 [arXiv: 1501.00568] [InSPIRE].

[73] H. Fujita, M. Nishida, M. Nozaki and Y. Sugimoto, Dynamics of logarithmic negativity and mutual information in smooth quenches, PTEP 2020 (2020) 073B02 [arXiv:1812.06258] [INSPIRE].

[74] J. Cardy, Thermalization and revivals after a quantum quench in conformal field theory, Phys. Rev. Lett. 112 (2014) 220401 [arXiv: 1403.3040] [INSPIRE].

[75] K. Najafi and M.A. Rajabpour, On the possibility of complete revivals after quantum quenches to a critical point, Phys. Rev. B 96 (2017) 014305 [arXiv:1707.07178] [INSPIRE].

[76] R. Modak, V. Alba and P. Calabrese, Entanglement revivals as a probe of scrambling in finite quantum systems, J. Stat. Mech. 2008 (2020) 083110 [arXiv:2004.08706] [INSPIRE].

[77] C.T. Asplund, A. Bernamonti, F. Galli and T. Hartman, Entanglement scrambling in $2 d$ conformal field theory, JHEP 09 (2015) 110 [arXiv:1506.03772] [INSPIRE].

[78] O. Guhne and G. Toth, Entanglement detection, Phys. Rept. 474 (2009) 1.

[79] A. Elben et al., Mixed-state entanglement from local randomized measurements, Phys. Rev. Lett. 125 (2020) 200501 [arXiv: 2007.06305] [INSPIRE].

[80] T. Yu and J.H. Eberly, Sudden death of entanglement, Sciences 323 (2009) 598 [arXiv:0910.1396]. 
[81] A. Ferraro, D. Cavalcanti, A. Garcia-Saez and A. Acin, Thermal bound entanglement in macroscopic systems and area law, Phys. Rev. Lett. 100 (2008) 080502 [arXiv:0804.4867] [INSPIRE].

[82] Y.K. Yazdi, Zero modes and entanglement entropy, JHEP 04 (2017) 140 [arXiv: 1608.04744] [INSPIRE].

[83] G. Di Giulio and E. Tonni, Subsystem complexity after a global quantum quench, JHEP 05 (2021) 022 [arXiv : 2102.02764] [InSPIRE]. 